\title{
Composites with Imperfect Interface
}

\author{
By Robert Lipton and Bogdan Vernescu \\ Department of Mathematical Sciences, Worcester Polytechnic Institute, \\ 100 Institute Rd., Worcester, MA 01609, U.S.A.
}

September 26, 1994

\begin{abstract}
New variational principles are introduced, describing the effective conductivity tensor for anisotropic two-phase heat conducting composites with interfacial surface resistance between phases. These principles reduce to those of Hashin and Shtrikman in the limit of vanishing surface resistance. The new principles allow for an unconstrained class of trial fields.

New bounds are derived on the effective heat conductivity tensor for an anisotropic two-phase composite with interfacial surface resistance between phases. The new upper bound is given in terms of the two-point correlation function, component volume fractions and moment of inertia tensor for the surface of each heterogeneity. The new lower bound is given in terms of the interfacial surface area, component volume fractions and a scale free matrix of parameters. This matrix corresponds to the effective conductivity associated with the same geometry but with nonconducting inclusions.

The bounds are applied to theoretically predict the occurence of size effect phenomena in the effective heat transport behaviour of two-phase suspensions with interfacial resistance between phases. For monodisperse suspension of spheres a critical radius is found for which the effective heat conductivity equals that of the matrix. For polydisperse suspensions of spheres it is shown that, when the mean radius lies below the critical value, the effective conductivity lies below that of the matrix.
\end{abstract}

\section{Variational Principles}

\section{I.1 Introduction}

Composites of technological and physical interest often exhibit imperfect contact between constituent phases. Here we address the problem of estimating the effective thermal conductivity for composites with an interfacial resistance. Such resistance in 
composite materials may arise from the presence of impurities at phase boundaries. These impurities are due to oxide films or bonding agents at the phase interface.

Starting with the efforts of Maxwell (1904) and Rayleigh (1892) a great part of the literature has focused on the idealized case of perfect contact. Here one assumes the continuity of the temperature and heat flux across the phase interface. On the other hand, imperfect interfaces are described by discontinuous temperature fields. The jump in temperature is assumed to be proportional to the heat flux across the interface.

For composites with perfect contact, the variational description of effective properties have proved successful in the estimation of effective properties. One of the best known are the Hashin-Shtrikman variational principles (1962). These principles yield the celebrated Hashin-Shtrikman bounds for isotropic heat conducting composites made from two heat conductors specified by the conductivities $\sigma_{2}$ and $\sigma_{1}$ in proportions $\theta_{2}$ and $\theta_{1}$. The Hashin-Shtrikman bounds are the best possible in that they are attainable by special microgeometries, (see Hashin and Shtrikman, 1962). For anisotropic composites the Hashin-Shtrikman variational principle yields bounds in terms of the two point correlation functions, (see Willis (1982), Avellaneda and Milton (1989)).

In this paper we treat two phase composites with interfacial barrier conductivity specified by $\beta$. We assume that the composite is made from isotropic conductors specified by $\sigma_{2}>\sigma_{1}$ in the proportions $\theta_{2}, \theta_{1}$ respectively. Our tool is a new set of variational principles describing the effective heat conducting properties of anisotropic conductors with barrier resistance, see Section 2 equations (??) and (??).

We develop a systematic method that we refer to as the interface comparison method, to obtain the new variational formulation for the effective conductivity. The advantage of the new formulation is that the solution of the associated field equations involves fields that are not coupled at the two-phase boundary. Most importantly the solution operators for these problems have an explicit form or can be written in terms of solution operators for the perfect contact problem (see Section 2 of this paper and Sections 2 and 3 of Part II). We apply these principles to obtain new upper and lower bounds on the effective conductivity for anisotropic particulate composites with interfacial barrier resistance. These bounds are derived and presented in the second paper, see equations (II2.9) and (II3.35).

The lower bounds depend explicitly upon interfacial surface area, interfacial barrier conductivity, component conductivities, and volume fraction. In addition the 
bound includes a scale-free matrix of parameters. This matrix corresponds to the effective conductivity of a composite with nonconducting inclusions having the same geometry as the original composite.

For isotropic particulate mixtures with barrier resistance we can say more. We introduce the effective conductivity function associated with the same inclusion geometry but with perfect contact between phases. The poles and zeros of this function are confined to an interval $\left[L_{1}, L_{2}\right]$ on the negative real axis, see Bergman (1978) Golden and Papanicolaou (1983). Using the results of Bruno (1991) for the perfect contact case we are able to write lower bounds on the effective conductivity for composites with imperfect contact in terms of the data $L_{1}, L_{2}$, see II, Section 2.1, equation (??).

For isotropic monodisperse suspensions of spheres we show how to apply the estimates of Torquato and Rubinstein (1991) for the perfect contact case to obtain lower bounds for imperfectly bonded conductors in terms of the nearest neighbor distribution function, see II equation (??).

We present an upperbound for anisotropic composites in terms of volume fraction, the two point correlation function and the moment of inertia tensors of the particle surfaces, see II equation (3.35). For the special case of isotropic particulate composites we display upper bounds for particles of conductivity $\sigma_{2}$ (or $\sigma_{1}$ ) in a matrix of $\sigma_{1}$ (or $\left.\sigma_{2}\right)$. Our bounds are in terms of volume fraction and a parameter $\alpha$ given by the sum of polar moments of inertia of the surface of each particle see (II3.37).

In Part III we consider the behavior of the bounds in the surface area $s$ and total polar moment of inertia of the interface $\alpha$. We find that in the limits $s \rightarrow$ $\infty, \alpha \rightarrow 0$ that these bounds are linked to the effective properties of suspensions of nonconducting inclusions (III, Section 3).

We consider monodisperse suspensions of spheres of conductivity $\sigma_{2}$ in a matrix of $\sigma_{1}$ with $\sigma_{2}>\sigma_{1}$. For this case we exhibit a critical radius, $R_{c r}=\beta^{-1} \sigma_{2} \sigma_{1}\left(\sigma_{2}-\sigma_{1}\right)^{-1}$, for which the effective conductivity equals that of the matrix, (III, Section 4). This is used to establish optimality of upper and lower bounds on the effective conductivity for certain values of the geometric parameters $s$ and $\alpha$, (III, Section 5). For polydisperse suspensions of spheres we use the bounds to give new theoretical predictions of size effect phenomena. Indeed, it is shown that if the average sphere radius $\langle r\rangle$ is less than $R_{c r}$, then the effective conductivity lies below the matrix, (see III, Theorem (6.1)). We consider suspensions of particles of conductivity $\sigma_{2}$ in a matrix of $\sigma_{1}$ where $\sigma_{2}>\sigma_{1}$. Noting that the lower bound is monotone in the interfacial surface area at fixed volume fraction, we show that the 
effective conductivity is greater than the matrix provided the interfacial surface area is less than the value $d \theta_{2}^{-1} R_{c r}$, (see III Theorem 7.1) were $d=2,3$ and denotes the dimension. We present a necessary condition for the optimal design of an isotropic polydisperse suspension of spheres that maximizes the heat transport at fixed sphere volume fraction, see Part III Theorem 7.2. For isotropic monodisperse suspensions of spheres we can say more. Indeed, collecting our results (see III Sections 5,6, and 7) we note that: (1) for spheres of radius less than $R_{c r}$ the effective property lies below that of the matrix. (2) For spheres of radius greater than $R_{c r}$ the effective property lies strictly above the matrix, see III, Theorem 7.3.

In Part III, Theorems 8.1, 8.2 and 8.3 we use the monotonicity of the upper and lower bounds with respect to $\beta, s$, and $\alpha$ to obtain information on the composite geometry or interfacial surface resistance from measured values of the effective conductivity.

In the present paper we introduce and apply the interface comparison method to obtain new variational principles for composites with interfacial barrier resistance. The advantage of these new principles are that no supplementary constraints are imposed on the trial functions and that simple trial functions provide new bounds that are in some cases optimal, see (III, Section 5).

The equations of equilibrium for composites with interfacial barrier resistance as given in Section 2 (??- ??) involve the coupling of heat flux with a jump in temperature across the phase interface. Our new principles are designed to encode this coupling while at the same time allowing for an unconstrained class of trial fields. The choice of trials for this principle is a product space of fields; given by the space of periodic square integrable fields over the domain and by the space of square integrable functions defined on the phase boundary.

We conclude this part by illustrating the functional dependence of the effective heat conductivity on the barrier conductivity $\beta$. We show that the effective conductivity is monotonic in $\beta$ for fixed microgeometry. For fixed values of $\beta$ the effective conductivity is seen to be bounded above by that of a perfectly bonded composite and below by a composite with nonconducting inclusions. In this way we show that the effective conductivity for an isotropic mixture with interface resistance is bounded above by the Hashin-Shtrikman bound for composites with perfect contact. In part II we display bounds that improve upon these see (II, Section 4).

Lastly we note that the scope of this paper is not limited to the context of heat conductivity. Indeed, this problem is mathematically analogous to the problems of es- 
timating effective diffusivity for multiphase composites separated by permeable membranes (Latour et. al. (1994)) as well as electrostatic problems in composites with interface resistance. We further remark that the techniques introduced here can readily be applied to the context of two-phase elastic mixtures with interfacial slip, see Lipton and Vernescu (1995).

\section{I.2 Variational Principles for Effective Conductivity}

\section{I.2.1 Mathematical and Physical background and Basic Variational Principles}

For periodic heat conducting composites we may decompose the temperature field $T$ into two parts, a periodic fluctuation $\tilde{\phi}$ and a linear part $\xi \cdot \chi$ such that $T=\tilde{\phi}+\xi \cdot \chi$. Following Benveniste and Miloh (1986) the average intensity $\langle\nabla T\rangle$ seen by an "outside observer" is

$$
\langle\nabla T\rangle=\int_{\partial Q}(\tilde{\phi}+\xi \cdot x) n d s=\xi
$$

Here $Q$ is the unit cell occupied by the composite, $\partial Q$ is the boundary of the cell and $n$ is the outward directed normal.

The cell $Q$ is composed of two isotropic conducting materials occupying regions $Y_{1}$ and $Y_{2}$ separated by an interface denoted by $\Gamma$. The temperature inside the composite satisfies:

$$
\begin{gathered}
\nabla \cdot\left(\sigma_{i}(\nabla \tilde{\phi}+\xi)\right)=0 \text { in } Y_{i}, \quad i=1,2, \\
{[\sigma(x)(\nabla \tilde{\phi}+\xi)] \cdot n=0 \text { on } \Gamma,}
\end{gathered}
$$

and

$$
\sigma_{2}(\nabla \tilde{\phi}+\xi) \cdot n=-\beta[\tilde{\phi}]_{1}^{2} \text {, on } \Gamma
$$

Here $n$ denotes the normal to $\Gamma$ and points into the interior of phase- 1 and (??) is the continuity of the normal flux across $\Gamma$. Equation (??) represents the effect of thermal surface resistance. The quantity $\sigma_{2}(\nabla \tilde{\phi}+\xi) \cdot n$ in (??) is evaluated on the phase- 2 side of $\Gamma$. The solution of problem (??)-(??) is unique up to a constant; this is an application of the Lax-Milgram lemma. 
The positive parameter $\beta^{-1}$ represents the thermal barrier resistance. The $\beta=\infty$ limit corresponds to the case of perfect contact and condition (??) is replaced with $[\tilde{\phi}]=0$. The $\beta=0$ limit corresponds to a perfectly insulating surface and is usually referred to as the "adiabatic" boundary condition.

The effective conductivity tensor for the composite is defined by

$$
\sigma^{e} \xi=\int_{Q} \sigma(x)(\nabla \tilde{\phi}+\xi) d x
$$

The effective conductivity admits two variational formulations analogous to the Dirichlet and Thompson variational principles for the case of perfect contact. The first is:

$$
\sigma^{e} \xi \cdot \xi=\min _{\phi \in V}\left\{\int_{Q} \sigma(x)(\nabla \phi+\xi)^{2} d x+\beta \int_{\Gamma}([\phi])^{2} d s\right\}
$$

where the space $V$ consists of all square integrable, Q-periodic functions $\phi$ such that $\nabla \phi$ is square integrable in each phase. We note that the space $V$ allows for fields $\phi$ that are discontinuous across $\Gamma$.

The second variational principle is:

$$
\left(\sigma^{e}\right)^{-1} \bar{\tau} \cdot \bar{\tau}=\min _{\tau \in W}\left\{\int_{Q} \sigma^{-1}(x)|(\tau+\bar{\tau})|^{2} d x+\frac{1}{\beta} \int_{\Gamma}((\tau+\bar{\tau}) \cdot n)^{2} d s\right\}
$$

where the space $W$ is the infinite dimensional space of all $\mathrm{Q}$ periodic square integrable fields $\tau(x)$ characterized by

$$
\nabla \cdot \tau=0, \int_{Q} \tau d x=0, \text { and }[\tau] \cdot n=0 \text { on } \Gamma,
$$

and $\bar{\tau}$ is any constant vector. For similar variational principles for effective tensors of heterogeneous elastic materials with imperfect interface, see Lene and Leguillon (1982) and Hashin (1992).

\section{I.2.2 Interface Comparison Method Variational Principles}

We present two new variational principles describing the effective conductivity tensor.

Before stating the first variational principle we introduce a comparison material with conductivity $\gamma<\sigma_{1}$ and formulate two auxiliary conductivity problems. For a square integrable Q-periodic field $p$, the potential $\phi^{p}$ is a solution of:

$$
\begin{gathered}
\gamma \Delta \phi^{p}=-\operatorname{divp} \text { in } Y_{i}, \quad i=1,2, \\
{\left[p+\gamma \nabla \phi^{p}\right] \cdot n=0 \text { on } \Gamma,}
\end{gathered}
$$


and

$$
\left(p+\gamma \nabla \phi^{p}\right) \cdot n=0 \text { on } \Gamma \text {. }
$$

For a square integrable function $v$ defined on $\Gamma$ the potential $\phi^{v}$ is a solution of

$$
\begin{gathered}
\Delta \phi^{v}=0 \text { in } Y_{i}, \quad i=1,2, \\
{\left[\gamma \nabla \phi^{v}\right] \cdot n=0, \text { on } \Gamma,}
\end{gathered}
$$

and

$$
\gamma \nabla \phi^{v} \cdot n=-v \text { on } \Gamma
$$

We observe that the boundary value problems given by (??) - (??) and (??) - (??) can be separately solved in each phase region.

Introducing the linear operators $M$ and $R$ given by

$$
M p=\nabla \phi^{p} \text { in } Y_{1} \cup Y_{2}
$$

and

$$
R v=\nabla \phi^{v} \text { in } Y_{1} \cup Y_{2}
$$

one has the new variational principle given by:

$$
(P 1): \quad\left(\sigma^{e}-\gamma I+\frac{\gamma^{2}}{\beta} \int_{\Gamma} n \otimes n\right) \xi \cdot \xi=\max _{(p, v) \in \mathcal{P}}\{2 \underline{L}(\xi, p, v)-\underline{Q}(p, v)\}
$$

where the space $\mathcal{P}$ defines a couple $(p, v)$ of admissible bulk and surface polarizations $(p, v)$ where $p$ is square integrable and Q-periodic and $v$ is square integrable on $\Gamma$. The linear form $\underline{L}(\xi, p, v)$ is defined by

$$
\underline{L}(\xi, p, v)=\frac{\gamma}{\beta} \int_{\Gamma} v \xi \cdot n d s+\int_{Q}(p \cdot \xi) d x .
$$

The quadratic form $\underline{Q}(p, v)$ is given by

$$
\underline{Q}(p, v)=\int_{Q}(\sigma-\gamma)^{-1}|p|^{2} d x+\frac{1}{\beta} \int_{\Gamma} v^{2} d s+\gamma \int_{Q}|M p+R v|^{2} d x
$$

For the second new variational principle we introduce a comparison material with conductivity $\gamma>\sigma_{2}$ and formulate two auxiliary conductivity problems. For a square integrable Q-periodic field $p$ the potential $\psi^{p}$ is a solution of

$$
\begin{aligned}
& \Delta \psi^{p}=\text { divp in } Y_{1} \cup Y_{2} \\
& {\left[\nabla \psi^{p}-p\right] \cdot n=0 \text { on } \Gamma}
\end{aligned}
$$


and

$$
\left[\psi^{p}\right]=0 \text { on } \Gamma
$$

For a square integrable function $v$ defined on $\Gamma$, the potential $\psi^{v}$ is a solution of

$$
\begin{gathered}
\Delta \psi^{v}=0 \text { in } Y_{1} \cup Y_{2}, \\
{\left[\partial_{n} \psi^{v}\right]=0 \text { on } \Gamma,}
\end{gathered}
$$

and

$$
\left[\psi^{\nu}\right]=-v \text { on } \Gamma
$$

Introducing the linear operators $N$ and $S$ defined by

$$
N p=\nabla \psi^{p} \text { in } Q
$$

and

$$
S v=\nabla \psi^{v} \text { in } Y_{1} \cup Y_{2},
$$

one has:

$$
(P 2): \quad\left(\sigma^{e^{-1}}-\gamma^{-1} I\right) \bar{\tau} \cdot \bar{\tau}=\max _{(p, v) \in \mathcal{P}}\{2 \bar{L}(\bar{\tau}, p, v)-\bar{Q}(p, v)\}
$$

Here the linear form $\bar{L}(\bar{\tau}, p, v)$ is defined by

$$
\bar{L}(\bar{\tau}, p, v)=\int_{Q} p \cdot \bar{\tau}+\int_{\Gamma}(\bar{\tau} \cdot n) v d s
$$

and the quadratic form $\bar{Q}$ is given by

$$
\begin{aligned}
\bar{Q}(p, v) & =\int_{Q}\left(\sigma^{-1}-\gamma^{-1}\right)^{-1}|p|^{2} d x+\beta \int_{\Gamma}|v|^{2} d s \\
& +\gamma \int_{Q}\left|(N p+S v-p)-\int_{Q}(N p+S v-p) d y\right|^{2} d x
\end{aligned}
$$

Here we remark that the operator $N$ can be identified with the projection of square integrable periodic fields onto $Q$ periodic curl-free fields.

The right hand sides of (??) and (??) can be written alternatively as min max principles for appropriately chosen lagrangians. Doing so yields the following characterizations of the effective conductivity tensor:

$$
\begin{aligned}
\left(\sigma^{e}-\gamma I\right. & \left.+\frac{\gamma^{2}}{\beta} \int_{\Gamma} n \otimes n d s\right) \xi \cdot \xi=\max _{(p, v) \in \mathcal{P}} \min _{\phi \in V} \mathcal{L}(p, v, \phi) \\
& =\min _{\phi \in V} \max _{(p, v) \in \mathcal{P}} \mathcal{L}(p, v, \phi)
\end{aligned}
$$




$$
\begin{aligned}
\left(\sigma^{e^{-1}}\right. & \left.-\gamma^{-1} I\right) \bar{\tau} \cdot \bar{\tau}=\max _{(p, v) \epsilon \mathcal{P}} \min _{\tau \in W} \overline{\mathcal{L}}(p, v, \tau) \\
& =\min _{\tau \in W} \max _{(p, v) \in \mathcal{P}} \overline{\mathcal{L}}(p, v, \tau)
\end{aligned}
$$

where the convex-concave lagrangians $\underline{\mathcal{L}}$ and $\overline{\mathcal{L}}$ are given by

$$
\begin{aligned}
\underline{\mathcal{L}}(p, v, \phi) & \equiv 2 \int_{Q} p \cdot \xi d x+2 \frac{\gamma}{\beta} \int_{\Gamma} v \xi \cdot n d s-\int_{Q}(\sigma-\gamma)^{-1}|p|^{2} d x-\frac{1}{\beta} \int v^{2} d s \\
& +2 \int_{Q} p \cdot \nabla \phi d x+2 \int_{\Gamma} v[\phi] d s+\gamma \int_{Q}|\nabla \phi|^{2} d x
\end{aligned}
$$

and

$$
\begin{aligned}
\overline{\mathcal{L}}(p, v, \tau) & =2 \int_{Q} p \cdot \bar{\tau} d x+2 \int_{\Gamma} v(\bar{\tau} \cdot n) d s-\int_{Q}\left(\sigma^{-1}-\gamma^{-1}\right)^{-1}|p|^{2} d x-\beta \int_{\Gamma} v^{2} d s \\
& +2 \int_{Q} p \cdot \tau d x+2 \int_{\Gamma}(\tau \cdot n) v d s+\gamma^{-1} \int_{Q}|\tau|^{2} d x .
\end{aligned}
$$

We recall that the limit $\beta \rightarrow \infty$ corresponds physically to the perfect contact case in which there is no surface resistance. For this case the new variational principles (??) and (??) reduce to the well known Hashin-Shtrikman variational principles (Hashin and Shtrikman 1962) for conductors with perfect contact.

To show that (??) reduces to the lower Hashin-Shtrikman principle as $\beta \rightarrow \infty$ we appeal to the equivalent saddle formulation (??). Passing to the limit $\beta=\infty$ in (??) yields

$$
\begin{aligned}
\left(\sigma^{e}-\gamma I\right) \xi \cdot \xi & =\min _{\phi \in V} \max _{(p, v) \in \mathcal{P}}\left\{2 \int_{Q} p \cdot \xi d x-\int_{Q}(\sigma-\gamma)^{-1}|p|^{2} d x\right. \\
& \left.+2 \int_{Q} p \cdot \nabla \phi d x+2 \int_{\Gamma} v[\phi] d s+\gamma \int_{Q}|\nabla \phi|^{2} d x\right\} .
\end{aligned}
$$

We observe from the (??) that the minimum is obtained for $[\phi]=0$. Switching the order of max and min leaves the righthand side of (??) unchanged and one obtains:

$$
\begin{aligned}
\left(\sigma^{e}-\gamma I\right) \xi \cdot \xi & =\max _{p}\left\{2 \int_{Q} p \cdot \xi d x-\int_{Q}(\sigma-\gamma)|p|^{2} d x\right. \\
& \left.+\min _{\substack{\phi \in V \\
[\phi]=0}}\left\{2 \int_{Q} p \cdot \nabla \phi d x+\gamma \int_{Q}(\nabla \phi)^{2} d x\right\}\right\}
\end{aligned}
$$

for $\gamma<\sigma_{1}$ which is the lower Hashin-Shtrikman variational principle for two-phase conductors with perfect contact. Passing to the $\beta=\infty$ limit in (??) forces $v=0$ and we obtain:

$$
\begin{aligned}
\left(\sigma^{e^{-1}}-\gamma^{-1} I\right) \bar{\tau} \cdot \bar{\tau} & =\max _{p}\left\{2 \int_{Q} p \cdot \bar{\tau}-\int_{Q}\left(\sigma^{-1}-\gamma^{-1}\right)^{-1}|p|^{2} d x\right. \\
& \left.+\gamma^{-1} \int_{Q}\left|(N p-p)-\int_{Q}(N p-p) d y\right|^{2} d x\right\}
\end{aligned}
$$


for $\gamma>\sigma_{2}$ which is the upper Hashin-Shtrikman variational principle for the case of perfect contact. We note that the above statements can be made rigorous by appealing to the theory of epi/hypo-convergence introduced by Attouch and Wets (1983)

\section{I.3 Derivation of the variational principles}

\section{I.3.1 Lower variational principle by the interface comparison method}

In this section we derive the lower variational principle (P1) for the choice of isotropic comparison material with conductivity $\gamma<\sigma_{1}$. The sequence of steps outlined below comprise the interface comparison method. We begin with the variational principle (??). Noting that the solution $\tilde{\phi}$ of (??)-(??) is the minimizer of (??) we write:

$$
\sigma^{e} \xi \cdot \xi=\int_{Q} \sigma(x)|\nabla \tilde{\phi}+\xi|^{2} d x+\beta \int_{\Gamma}([\tilde{\phi}])^{2} d s .
$$

Adding and subtracting the reference energy $\gamma|\nabla \tilde{\phi}+\xi|^{2}$ to the right-hand side of (??) and rearrangement gives:

$$
\begin{aligned}
\left(\sigma^{e}-\gamma\right) \xi \cdot \xi & =\int_{Q}(\sigma(x)-\gamma)|\nabla \tilde{\phi}+\xi|^{2} d x+\gamma \int_{Q}|\nabla \tilde{\phi}|^{2} d x \\
& +2 \gamma \int_{Q} \nabla \tilde{\phi} \cdot \xi d x+\beta \int_{\Gamma}([\tilde{\phi}])^{2} d s
\end{aligned}
$$

Integrating by parts, one obtains

$$
2 \gamma \int_{Q} \nabla \tilde{\phi} \cdot \xi d x=2 \gamma \int_{\Gamma}[\tilde{\phi}] n \cdot \xi d s
$$

Applying (??) and completing the square in the last two terms of (??) gives

$$
\begin{aligned}
\left(\sigma^{e}-\gamma I+\frac{\gamma^{2}}{\beta} \int_{\Gamma} n \otimes n d s\right) \xi \cdot \xi & =\int_{Q}(\sigma(x)-\gamma)|\nabla \tilde{\phi}+\xi|^{2} d x+\gamma \int_{Q}|\nabla \tilde{\phi}|^{2} d x \\
& +\beta \int_{\Gamma}\left([\tilde{\phi}]_{+}+\frac{\gamma}{\beta} \xi \cdot n\right)^{2} d s .
\end{aligned}
$$

Introducing the bulk and surface polarizations $p$ and $v$ one has the elementary estimates:

$$
\beta \int_{\Gamma}\left([\tilde{\phi}]+\frac{\gamma}{\beta} \xi \cdot n\right)^{2} d s \geq \int_{\Gamma} 2 v\left([\tilde{\phi}]+\frac{\gamma}{\beta} \xi \cdot n\right) d s-\frac{1}{\beta} \int_{\Gamma} v^{2} d s
$$

and

$$
\int_{Q}(\sigma(x)-\gamma)|\nabla \tilde{\phi}+\xi|^{2} d x \geq 2 \int_{Q} p \cdot(\nabla \tilde{\phi}+\xi) d x-\int_{Q}(\sigma(x)-\gamma)^{-1}|p|^{2} d x
$$


for any square integrable and Q-periodic field $p$ and any function $v$ square integrable on $\Gamma$. We denote the set of all pairs $(p, v)$ by $\mathcal{P}$. Applying estimates (??) and (??) to (??) we obtain

$$
\left(\sigma^{e}-\gamma I+\frac{\gamma^{2}}{\beta} \int_{\Gamma} n \otimes n d s\right) \xi \cdot \xi \geq \underline{\mathcal{L}}(p, v, \tilde{\phi})
$$

where $\underline{\mathcal{L}}$ is defined by (??). Next we observe

$$
\left(\sigma^{e}-\gamma I+\frac{\gamma^{2}}{\beta} \int_{\Gamma} n \otimes n d s\right) \xi \cdot \xi \geq \underline{\mathcal{L}}(p, v, \tilde{\phi}) \geq \inf _{\phi \in V} \underline{\mathcal{L}}(p, v, \phi)=\underline{\mathcal{L}}\left(p, v,{ }_{\phi}^{*}\right)
$$

where $\stackrel{*}{\phi}$ is the minimizer of:

$$
\inf _{\phi \in V}\left\{2 \int_{Q} \nabla \phi \cdot p d x+2 \int_{\Gamma} v[\phi]+\gamma \int_{Q}|\nabla \phi|^{2} d x\right\}
$$

and satisfies

$$
\begin{gathered}
-\gamma \Delta \stackrel{*}{\phi}=\operatorname{divp} \text { in } Y_{1} \cup Y_{2}, \\
{[\gamma \nabla \stackrel{*}{\phi}+p] \cdot n=0 \text { on } \Gamma,}
\end{gathered}
$$

and

$$
(\gamma \nabla \stackrel{*}{\phi}+p) \cdot n=-v \text { on } \Gamma
$$

Observing that $\stackrel{*}{\phi}$ is linear in the data $(p, v)$ we write $\stackrel{*}{\phi}=\phi^{p}+\phi^{v}$ where $\phi^{p}$ and $\phi^{v}$ solve problems (??)-(??) and (??)-(??) respectively. Recalling the definitions of the operators $M$ and $R$ given by (??) and (??), inequality (??) can be written as the variational inequality:

$$
\left(\sigma^{e}-\gamma I+\frac{\gamma^{2}}{\beta} \int_{\Gamma} n \otimes n d s\right) \xi \cdot \xi \geq 2 \underline{L}(\xi, p, v)-\underline{Q}(p, v)
$$

One observes for the choice of bulk and surface polarizations, consistent with the actual potential inside the composite, i.e.,

$$
p=(\sigma-\gamma)(\nabla \tilde{\phi}+\xi) \text { and } v=\beta\left([\tilde{\phi}]+\frac{\gamma}{\beta} \xi \cdot n\right)
$$

that (??) holds with equality. Thus we have established ( $P 1)$. Additionally it follows from (??) and the previous observation that

$$
\left(\sigma^{e}-\gamma I+\frac{\gamma^{2}}{\beta} \int_{\Gamma} n \otimes n d s\right) \xi \cdot \xi=\max _{(p, v) \in \mathcal{P}} \min _{\phi \in V} \underline{\mathcal{L}}(p, v, \phi)
$$

and equation (??) follows. 


\section{I.3.2 Upper variational principle by the interface comparison method}

Here we establish (P2) for the choice of isotropic comparison material with conduc-

tivity $\gamma>\sigma_{2}$. We consider the variational principle (??) and denote the minimizer by $\tilde{\tau}$. The effective energy is written:

$$
\left(\sigma^{e}\right)^{-1} \bar{\tau} \cdot \bar{\tau}=\int_{Q} \sigma^{-1}(x)|\tilde{\tau}+\bar{\tau}|^{2} d x+\frac{1}{\beta} \int_{\Gamma}((\tilde{\tau}+\bar{\tau}) \cdot n)^{2} d s .
$$

Adding and subtracting the reference energy $\gamma^{-1}|\tilde{\tau}+\bar{\tau}|^{2}$ to the right-hand side of (??) yields:

$\left(\sigma^{e^{-1}}-\gamma^{-1}\right) \bar{\tau} \cdot \bar{\tau}=\int_{Q}\left(\sigma^{-1}(x)-\gamma^{-1}\right)|\tilde{\tau}+\bar{\tau}|^{2} d x+\int_{Q} \gamma^{-1}|\tilde{\tau}|^{2} d x+\frac{1}{\beta} \int_{\Gamma}((\tilde{\tau}+\bar{\tau}) \cdot n)^{2} d s$.

One has the elementary estimates:

$$
\int_{Q}\left(\sigma^{-1}(x)-\gamma^{-1}\right)|\tilde{\tau}+\bar{\tau}|^{2} d x \geq 2 \int_{Q} p \cdot(\tilde{\tau}+\bar{\tau}) d x-\int_{Q}\left(\sigma^{-1}(x)-\gamma^{-1}\right)^{-1}|p|^{2} d x
$$

and

$$
\frac{1}{\beta} \int_{\Gamma}((\tilde{\tau}+\bar{\tau}) \cdot n)^{2} d s \geq 2 \int_{\Gamma}(\tilde{\tau}+\bar{\tau}) \cdot n v d s-\int_{\Gamma} \beta v^{2} d s,
$$

for any square integrable Q-periodic field $p$ and any square integrable function $v$ on $\Gamma$.

Application of the estimates to (??) yield:

$$
\left(\sigma^{e^{-1}}-\gamma^{-1}\right) \bar{\tau} \cdot \bar{\tau} \geq \overline{\mathcal{L}}(p, v, \tilde{\tau}),
$$

where $\overline{\mathcal{L}}$ is defined by (??). Now we observe that

$$
\left(\sigma^{e^{-1}}-\gamma^{-1}\right) \bar{\tau} \cdot \bar{\tau} \geq \overline{\mathcal{L}}(p, v, \tilde{\tau}) \geq \inf _{\tau \in W} \overline{\mathcal{L}}(p, v, \tau)=\overline{\mathcal{L}}(p, v, \stackrel{*}{\tau})
$$

where $\stackrel{*}{\tau}$ is the minimizer of:

$$
\inf _{\tau \in W}\left\{2 \int_{Q} p \cdot \tau d x+2 \int_{\Gamma} \tau \cdot n v d s+\gamma^{-1} \int_{Q}|\tau|^{2} d x\right\}
$$

Calculation shows that $\stackrel{*}{\tau}$ is given by

$$
\stackrel{*}{\tau}=\gamma\left\{\nabla \stackrel{*}{\psi}-p-\int_{Q}(\nabla \stackrel{*}{\psi}-p) d x\right\}
$$

where $\stackrel{*}{\psi}$ is the solution of:

$$
\Delta \stackrel{*}{\psi}=\operatorname{divp} \text { in } Y_{1} \cup Y_{2},
$$




$$
[\nabla \stackrel{*}{\psi}-p] \cdot n=0 \text { on } \Gamma
$$

and

$$
[\stackrel{*}{\psi}]=-v \text { on } \Gamma \text {. }
$$

Noting that $\stackrel{*}{\psi}$ is linear in the data $(p, v)$ we write $\stackrel{*}{\psi}=\psi^{p}+\psi^{v}$ where $\psi^{p}$ and $\psi^{v}$ solve problems (??) - (??) and (??) - (??) respectively. Recalling the definition for the operators $N$ and $S$ given by (??) and (??), inequality (??) can be written as the variational inequality.

$$
\left(\sigma^{e^{-1}}-\gamma^{-1}\right) \bar{\tau} \cdot \bar{\tau} \geq 2 \bar{L}(\bar{\tau}, p, v)-\bar{Q}(p, v) .
$$

For the choice of bulk and surface polarizations, consistent with the actual heat flux $\tilde{\tau}+\bar{\tau}$ inside the composite, i.e.,

$$
p=\left(\sigma^{-1}(x)-\gamma^{-1}\right)(\tilde{\tau}+\bar{\tau}), \quad v=\frac{1}{\beta}(\tilde{\tau}+\bar{\tau}) \cdot n
$$

one observes that (??) holds with equality. In this way we arrive at (P2). Additionally it follows from (??) and the previous observation that

$$
\left(\sigma^{e^{-1}}-\gamma^{-1}\right) \bar{\tau} \cdot \bar{\tau}=\max _{(p, v) \in \mathcal{P}} \min _{\tau \in W} \overline{\mathcal{L}}(p, v, \tau),
$$

and equation (??) follows.

\section{I.4 Dependence of the effective properties on the interfacial barrier resistance}

The effective conductivity is easily seen to be monotonic decreasing as a function of the interfacial barrier resistance $\beta^{-1}$. To reflect the dependence of the effective conductivity on the barrier resistance we denote the effective conductivity tensor of a possibly anisotropic composite by $\sigma^{e}(\beta)$. For particulate composites we let $\sigma^{e}(0)$ denote the effective conductivity for particles made of nonconducting material, and $\sigma^{e}(\infty)$ denote the effective conductivity of a perfectly bonded composite with no interface resistance. For a fixed particle geometry we have the estimate

$$
\sigma^{e}(0) \leq \sigma^{e}(\beta) \leq \sigma^{e}(\infty) .
$$

For anisotropic composites the estimate holds in the sense of quadratic forms. With out loss of generality we suppose that the particle phase is occupied by material-2 and the matrix by material-1. We now establish this estimate. 
We start by showing $\sigma^{e}(0) \leq \sigma^{e}(\beta)$. Denoting the lagrangian on the righthand side of (??) by $G(\beta, \phi)$ we have

$$
\sigma^{e}(\beta) \xi \cdot \xi=\inf _{\phi \in V} G(\beta, \phi)
$$

From monotonicity we have:

$$
\inf _{\phi \in V} G(0, \phi) \leq \sigma^{e}(\beta) \xi \cdot \xi
$$

Next we show that $\sigma^{e}(0) \xi \cdot \xi=\inf _{\phi \in V} G(0, \phi)$.

We have:

$$
\inf _{\phi \in V} G(0, \phi)=\inf _{\phi \in V} \int_{Q} \sigma(x)|\nabla \phi+\xi|^{2} d x .
$$

The associated Euler equations are:

$$
\begin{gathered}
\Delta \phi=0 \text { in } Y_{1} \cup Y_{2} \\
\sigma_{1}(\nabla \phi+\xi)_{1} \cdot n=0 \text { on } \Gamma \\
\sigma_{2}(\nabla \phi+\xi)_{2} \cdot n=0 \text { on } \Gamma
\end{gathered}
$$

For the particle phase we find from (??) and (??) that $\phi=-\xi \cdot x$ in $Y_{2}$ thus $\nabla \phi+\xi=0$ identically in the particle. Equations (??) and (??) are those associated with heat conductivity exterior to a nonconducting inclusion and

$$
\inf _{\phi \in V} G(0, \phi)=\inf _{\phi \in V} \int_{Y_{1}} \sigma_{1}|\nabla \phi+\xi|^{2} d x=\sigma^{e}(0) \xi \cdot \xi
$$

Regarding the upper bound in (??), from monotonicity we have

$$
\sigma^{e}(\beta) \xi \cdot \xi \leq \inf _{\phi \in V} G(\infty, \phi)
$$

where $G(\infty, \phi)$ is given by

$$
G(\infty, \phi)=\int_{Q} \sigma(x)|\nabla \phi+\xi|^{2} d x+\left\{\begin{array}{l}
0,[\phi]=0 \\
\infty,[\phi] \neq 0
\end{array}\right.
$$

Now we show that $\sigma^{e}(\infty) \xi \cdot \xi=\inf _{\phi \in V} G(\infty, \phi)$. From (??) we see that the infimum is carried out over all functions continuous across the phase interface, i.e.,

$$
\inf _{\phi \in V} G(\infty, \phi)=\inf _{\substack{\phi \in V \\[\phi]=0}} G(\infty, \phi)
$$


The resulting Euler equations are those for no thermal interface resistance, and are given by

$$
\begin{gathered}
\sigma_{i} \Delta \phi=0 \text { in } Y_{i}, \quad i=1,2 \\
{[\sigma(\nabla \phi+\xi)] \cdot n=0 \text { on } \Gamma}
\end{gathered}
$$

and

$$
[\phi]=0 .
$$

Thus

$$
\inf _{\phi \in V} G(\infty, \phi)=\sigma^{e}(\infty) \xi \cdot \xi
$$

We conclude by showing that the bounds in (??) are obtained by $\sigma^{e}(\beta)$ in the limits $\beta \searrow 0$ and $\beta \nearrow \infty$ respectively. Indeed, we show $\lim _{\beta \backslash 0} \sigma^{e}(\beta)=\sigma^{e}(0)$. First monotonicity gives:

$$
\sigma^{e}(0) \leq \lim _{\beta \backslash 0} \sigma^{e}(\beta)
$$

On the other hand we have the estimate

$$
\lim _{\beta \backslash 0} \sigma^{e}(\beta)=\lim _{\beta \backslash 0} \inf _{\phi} G(\beta, \phi) \leq \inf _{\phi} G(0, \phi)=\sigma^{e}(0),
$$

and the claim follows immediately from estimates (??) and (??). To show $\lim _{\beta / \infty} \sigma^{e}(\beta)=\sigma^{e}(\infty)$ we utilize the complementary principle (??). We write (??) as

$$
\sigma^{e^{-1}}(\beta) \xi \cdot \xi=\inf _{\tau \in W} F(\beta, \tau)
$$

For the choice $\beta=\infty$ it is easily seen using standard arguments, (c.f. Kohn, Milton (1988)) that

$$
\inf _{\tau \in W} F(\infty, \tau)=\sigma^{e^{-1}}(\infty) \bar{\tau} \cdot \bar{\tau}
$$

Monotonicity gives:

$$
\sigma^{e^{-1}}(\infty) \leq \lim _{\beta / \infty} \sigma^{e^{-1}}(\beta)
$$

One also has the estimate:

$$
\lim _{\beta \nearrow \infty} \sigma^{e^{-1}}(\beta) \bar{\tau} \cdot \bar{\tau}=\lim _{\beta \nearrow \infty} \inf _{\tau \in W} F(\beta, \tau) \leq \inf _{\tau \in W} F(\infty, \tau)=\sigma^{e^{-1}}(\infty) \bar{\tau} \cdot \bar{\tau},
$$

and from estimates (??) and (??) we obtain

$$
\lim _{\beta>\infty} \sigma^{e-1}(\beta)=\sigma^{e^{-1}}(\infty)
$$

or equivalently $\lim _{\beta>\infty} \sigma^{e}(\beta)=\sigma^{e}(\infty)$. 
We denote the Hashin-Shtrikman second order upper bounds (see, Kohn and Milton (1988)), anisotropic composites at fixed volume fraction by $\mathrm{HS}^{+}$. Thus $\sigma^{e}(\infty) \leq H S^{+}$and we have the elementary bounds on $\sigma^{e}(\beta)$ at fixed volume fraction given by

$$
\sigma^{e}(0) \leq \sigma^{e}(\beta) \leq H S^{+}
$$

In Part II we apply the variational principles to obtain new bounds improving on those appearing in (??). The reader is referred to equations (II4.4), (II4.5), and (II4.6) in Section 4.

\section{Bounds}

\section{II.1 Introduction}

Here, we make use of the new variational principles derived in Part I to obtain new bounds for the effective conductivity $\sigma^{\mathbf{e}}$ of a composite with interfacial surface resistance.

We consider composites in $\Re^{d}(d=2,3)$ made from two isotropic heat conductors with conductivities specified by $\sigma_{1}, \sigma_{2}$ where without loss of generality we take $\sigma_{2}>$ $\sigma_{1}$. The interfacial barrier is characterized by the thermal surface conductivity $\beta$. The interfacial surface area is denoted by $s$ and the volume fractions of the two conductors are denoted by $\theta_{1}$ and $\theta_{2}\left(\theta_{1}+\theta_{2}=1\right)$.

Bounds that use reduced microstructural information can be obtained from the basic variational principles given by eqs. (I2.6) and (I2.7) of Part I. Indeed choosing constant trial fields in (I2.6) and (I2.7) yields:

$$
\left(\frac{\theta_{1}}{\sigma_{1}}+\frac{\theta_{2}}{\sigma_{2}}+\frac{s}{d \beta}\right)^{-1} \mathbf{I} \leq \sigma^{e} \leq\left(\theta_{1} \sigma_{1}+\theta_{2} \sigma_{2}\right) \mathbf{I} .
$$

Non-constant trial fields that encode microstructural information naturally lead to more refined bounds. For the case of a monodisperse suspension of spheres of radius "a" and conductivity $\sigma_{2}$ in a matrix of conductivity $\sigma_{1}$, we choose trial fields of the form:

$$
\phi= \begin{cases}0 & \text { in the matrix } \\ y^{m} \cdot \eta & \text { in the mth sphere }\end{cases}
$$


here $y^{m}$ equals $x-r^{m}$, with $r^{m}$ being the center of the mth sphere. Restriction of trial fields in (I2.6) to the class given above and subsequent optimization over $\eta$ gives the upper bound:

$$
\sigma^{e} \leq\left[\theta_{1} \sigma_{1}+\theta_{2} \sigma_{2}\left(1-\frac{1}{1+\frac{\beta a}{\sigma^{2}}}\right)\right] I .
$$

Phase interchange yields the upper bound for monodisperse suspension of spheres of conductivity $\sigma_{1}$ in a matrix of $\sigma_{2}$. We remark that trial fields of the type given by (??) were previously introduced in the context of two-phase elastic composites with interfacial slip by Hahsin (1992).

Here we will present bounds that improve on (??) and (??) as well as the bounds given in Section 4 of Part I, see equation (I4.23). For certain parameter values we show that our bounds are optimal (see Part III, Section 5).

For anisotropic particulate composites we exhibit lower bounds in terms of volume fraction, interfacial surface area and a scale-free matrix of parameters. This matrix corresponds to the effective conductivity associated with the same particle geometry but with non-conducting particles (see (??)).

We specialize to isotropic suspensions of particles in a matrix phase. For suspensions of particles of conductivity $\sigma_{1}$ (or $\sigma_{2}$ ) in a matrix of $\sigma_{2}$ (or $\sigma_{1}$ ) we exhibit new bounds (see, (??) and (??)) that are strictly better than the lower bound in (??) as well as those presented in Section 4 of Part I, see equation (I4.23). These new bounds ((??) and (??)) provide the means to estimate the effective properties for composites with imperfect interface in terms of the effective conductivity function associated with perfectly bonded composites having no interfacial barrier resistance. It is well known that the poles and zeroes of this function are confined to the negative seal axis, see, Bergman (1978). When the poles and zeros are confined to an interval $\left[L_{1}, L_{2}\right]$ Bruno (1991) has derived lower bounds on the effective conductivity for the perfect contact case in terms of $L_{1}$ and $L_{2}$. We indicate how to use our new bounds and Bruno's estimates to recover a new lower bound for the effective conductivity for composites with interfacial surface resistance, in terms of the parameters $L_{1}, L_{2}$, (see eq. (??).

On the other hand, for a monodisperse distribution of spheres we apply the methods of Torquato and Rubinstein (1991) to bound the scale-free part of our bounds (??), (??) in terms of the nearest neighbor distribution function. This yields a new lower bound for monodisperse suspensions of spheres in the presence of interfacial surface resistance, see Section 2 equation (??). 
In Section 3 we present an upper bound for anisotropic composites in terms of volume fraction, the two point correlation function, and the moment of inertia tensors of the particle surfaces. To illustrate our method we focus on isotropic particulate composites and display upper bounds for particles of conductivity $\sigma_{2}$ (or $\left.\sigma_{1}\right)$ in a matrix of $\sigma_{1}$ (or $\sigma_{2}$ ). Our estimates are in terms of volume fraction and a parameter $\alpha$ given by the sum of polar moments of intertia of the surface of each particle, see $(? ?)$.

For monodisperse suspensions of spheres of conductivity $\sigma_{2}$ in a matrix of $\sigma_{1}$ our new upper bound is always tighter than the upper bound given by (??), see Section 4. We illustrate the relation between the new bounds derived in Section 3 with those obtained from simpler variational principles.

We remark that the new upper and lower bounds are sensitive to size effects. We postpone a full analysis of the size effect phenomena predicted by the upper and lower bounds until the third part.

\section{II.2 New lower bounds on the effective conductivity}

We apply variational principles developed in Part I to obtain lower bounds on the effective conductivity tensor. Here we obtain lower bounds on the effective conductivity for particulate composites in terms of volume fraction, surface area, and a scale free matrix of parameters. This matrix is the effective conductivity of a composite with the same microgeometry but non-conducting inclusions.

\section{II.2.1 Particles of high conductivity in a low conductivity matrix}

We consider particles of phase 2 embedded in a matrix of phase 1 with $\sigma_{2}>\sigma_{1}$. We suppose that none of the inclusions intersect the period cell boundary.

To obtain new lower bounds we make the specific choice of surface and bulk polarization fields, in the variational principle (P1) (equation I2.17) given by

$$
p=\chi_{2} \mu, v=r \cdot n
$$

where $\mu$, and $r$ are vectors in $\Re^{d}$ and $n$ is the normal pointing into phase 1 . The associated bound is given by

$$
\left(\sigma^{e}-\gamma \mathbf{I}+\frac{\gamma^{2}}{\beta} \int_{\Gamma} n \otimes n\right) \xi \cdot \xi \geq \max _{\substack{\mu \in \Re d \\ r \in \Re d}}\left\{2 \underline{L}\left(\xi, \chi_{2} \mu, r \cdot n\right)-\underline{Q}\left(\chi_{2} \mu, r \cdot n\right)\right\} .
$$


We now set the comparison conductivity $\gamma$ to $\sigma_{1}$ and present the explicit form of $\underline{L}$ and $Q$ :

$$
\begin{gathered}
\underline{L}=\frac{\sigma_{1}}{\beta} \int_{\Gamma} n \otimes n d s \xi \cdot r+\theta_{2} \xi \cdot \mu \\
\underline{Q}=\theta_{2}\left(\sigma_{2}-\sigma_{1}\right)^{-1}|\mu|^{2}+\frac{1}{\beta} \int_{\Gamma} n \otimes n d s r \cdot r \\
+\sigma_{1}^{-1}\left(\theta_{2}|\mu|^{2}+2 \theta_{2} \mu \cdot r+\left(\mathbf{I}-\mathbf{m}_{0}\right) r \cdot r\right)
\end{gathered}
$$

Here the symmetric $d \times d$ tensor $\mathbf{m}_{0}$ is the effective conductivity tensor of a composite with non-conducting particles having the same microgeometry as the original composite. The region $Y_{1}$ is assumed to be composed of an isotropic conductor with unit conductivity and $Y_{2}$ is filled with a perfect insulator. For any constant electric field $\xi$ the possibly anisotropic effective conductivity is defined by:

$$
\mathbf{m}_{0} \xi \cdot \xi=\inf _{\phi \in H_{p e r}^{1}} \int_{Y_{1}}|\nabla \phi+\xi|^{2} d x
$$

We observe that equation (??) for $\underline{L}$ and the first two terms in (??) follow directly upon substitution of the polarizations into (P1)(of I). The last three terms in (??) follow from solution of the comparison problems (I2.9)-(I2.11) and (I2.12)-(I2.14) and evaluation of the non-local term in (I2.19). Indeed, we have the;

Lemma II.2.1 For the choice $p=\chi_{2} \mu$ and $v=r \cdot n$, the nonlocal term $\int_{Q}|M p+R v|^{2} d x$ in (ID.19) is given by

$$
\sigma_{1}^{-2}\left(\theta_{2}|\mu|^{2}+2 \theta_{2} \mu \cdot r+\left(\mathbf{I}-\mathbf{m}_{0}\right) r \cdot r\right)
$$

Proof. For the choice $p=\chi_{2} \mu$, solution of (I2.9)-(I2.11), yields $M \chi_{2} \mu=-\sigma_{1}^{-1} \chi_{2} \mu$, and therefore:

$$
\int_{Q}|M p|^{2} d x=\theta_{2} \sigma_{1}^{-2}|\mu|^{2}
$$

Solution of (I2.12)-(I2.14) provides us with the relation $R(r \cdot n)=-\sigma_{1}^{-1} r$ in region-2 and so

$$
2 \int_{Q} M p \cdot R v d x=2 \theta_{2} \sigma_{1}^{-2} r \cdot \mu
$$

Last in region-1 we have that $R(r \cdot n)$ is given by $\nabla \phi^{r} \equiv \sum_{i=1}^{d} r_{i} \nabla \tilde{\phi}^{i}$ where $\tilde{\phi}^{i}$ is a solution of

$$
\Delta \tilde{\phi}^{i}=0 \text { in } Y_{1}
$$




$$
\left(\nabla \tilde{\phi}^{i}+\sigma_{1}^{-1} e^{i}\right) \cdot n=0 \text { on } \Gamma
$$

and $\tilde{\phi}^{i}$ periodic on $Q$. In this way we see that $\tilde{\phi}^{i}$ is the periodic fluctuation in the potential for a composite made from pure insulator in region- 2 and an isotropic conductor in region- 1 . Here the composite is subject to a constant gradient $\sigma_{1}^{-1} e^{i}$. The vector $e^{i}$ denotes the unit vector in the $\mathrm{i}$-th coordinate direction. Indeed, integration by parts and application of (??) and (??) yields

$$
\int_{Y_{1}}\left|\nabla \phi^{r}\right|^{2} d x+\sigma_{1}^{-1} \int_{Y_{1}} \nabla \phi^{r} \cdot r d x=0
$$

Completing squares gives:

$$
\sigma_{1}^{-2} \mathbf{m}_{0} r \cdot r \equiv \int_{Y_{1}}\left|\nabla \phi^{r}+\sigma_{1}^{-1} r\right|^{2} d x=\sigma_{1}^{-2} \theta_{1}|r|^{2}-\int_{Y_{1}}\left|\nabla \phi^{r}\right|^{2} d x .
$$

We observe for the choice $v=r \cdot n$, the term $\int_{Q}|R v|^{2} d x$ is given by:

$$
\int_{Y_{2}}|R v|^{2} d x+\int_{Y_{1}}|R v|^{2} d x=\theta_{2} \sigma_{1}^{-2}|r|^{2}+\int_{Y_{1}}\left|\nabla \phi^{r}\right|^{2} d x .
$$

Finally application of (??) gives

$$
\int_{Q}|R v|^{2} d x=\sigma_{1}^{-2}|r|^{2}-\sigma_{1}^{-2} \mathbf{m}_{0} r \cdot r
$$

Now we set $\frac{1}{\beta} \int_{\Gamma} n \otimes n d s=\mathbf{B}$ and maximize (??) over $\mu$ and $r$ in $\Re^{d}$ to obtain the lower bound on the effective conductivity given by:

$$
\begin{gathered}
\left(\sigma^{e}-\sigma_{1} \mathbf{I}+\sigma_{1}^{2} \mathbf{B}\right) \xi \cdot \xi \geq\left(\begin{array}{cc}
\theta_{2} \frac{\sigma_{2}}{\sigma_{1}}\left(\sigma_{2}-\sigma_{1}\right)^{-1} \mathbf{I} & \theta_{2} \sigma_{1}^{-1} \mathbf{I} \\
\theta_{2} \sigma_{1}^{-1} \mathbf{I} & \mathbf{B}+\sigma_{1}^{-1}\left(\mathbf{I}-\mathbf{m}_{0}\right)
\end{array}\right)^{-1} \\
\left(\begin{array}{c}
\theta_{2} \xi \\
\sigma_{1} \mathbf{B} \xi
\end{array}\right) \cdot\left(\begin{array}{c}
\theta_{2} \xi \\
\sigma_{1} \mathbf{B} \xi
\end{array}\right)
\end{gathered}
$$

This bound holds for all choices of constant temperature gradients $\xi$ in $\Re^{d}$. For isotropic mixtures the tensor $\mathbf{B}$ reduces to:

$$
\mathbf{B}=\frac{s}{d \beta} \mathbf{I}, \quad d=2,3,
$$

and inequality (??) becomes the lower bound on the effective conductivity for isotropic particulate composites given by:

$$
\sigma^{e} \geq \sigma_{1}-\sigma_{1}\left(\left(1-m_{0}\right)^{-1}+\left(\sigma_{1} \theta_{2} c\right)^{-1}\right)^{-1}
$$


where $c$ represents the characteristic combination

$$
c=\frac{s}{d \theta_{2} \beta}-\frac{\sigma_{2}-\sigma_{1}}{\sigma_{1} \sigma_{2}} .
$$

and $m_{0}$ is the scalar effective conductivity of a composite with the same geometry but with nonconducting inclusions in a matrix of unit conductivity.

We denote the lower bound on the right hand side of (??) by $I C L_{12}\left(m_{0}, \beta\right)$. Elementary Wiener bounds on $m_{0}$ show that this parameter is restricted to the interval $\left[0, \theta_{1}\right]$. Analysis shows that for $m_{0}$ fixed, $0<m_{0} \leq \theta_{1}$, and $\beta \geq 0$, one has $I C L_{12}\left(m_{0}, \beta\right)$ monotone increasing in $\beta$ and

$$
\sigma^{e} \geq I C L_{12}\left(m_{0}, \beta\right) \geq I C L_{12}\left(m_{0}, 0\right)=\sigma_{1} m_{0}
$$

Here for $\beta=0$ the bound reduces to $\sigma_{1} m_{0}$. From Bergman (1978) it is known that the effective conductivity is a homogeneous function of the component conductivities and so the quantity $\sigma_{1} m_{0}$ is precisely the effective conductivity for a matrix of conductivity $\sigma_{1}$ with insulating inclusions. On the other hand the bound $I C L_{12}\left(m_{0}, \beta\right)$ is found to be monotone increasing in $m_{0}$ for $\beta \geq 0$, and

$$
\sigma^{e} \geq I C L_{12}\left(m_{0}, \beta\right) \geq I C L_{12}(0, \beta)=\left(\frac{\theta_{1}}{\sigma_{1}}+\frac{\theta_{2}}{\sigma_{2}}+\frac{s}{d \beta}\right)^{-1} .
$$

Here $I C L_{12}(0, \beta)$ is the analogue of the Wiener lower bound for the case of perfect conductivity. The bound $I C L_{12}(0, \beta)$ may be obtained directly from variational principle (I2.7).

It is shown in the work of Bruno (1991) that effective transport properties for composites with nonconducting inclusions can be characterized in terms of the singularities and zeros of the effective conductivity function for perfectly bonded conductors. These zeroes and singularities are known to be confined to an interval on the negative real axis, see Bergman (1978). For composites with singularities and zeros lying inside an interval $\left[L_{1}, L_{2}\right]$ on the negative real axis we set:

$$
s_{m}=\frac{1}{1-L_{1}} \text { and } S_{m}=\frac{1}{1-L_{2}} .
$$

From the work of Bruno (1991) it follows that the conductivity $m_{0}$ is bounded below by

$$
m_{0} \geq K\left(s_{m}, S_{m}, \theta_{2}\right)
$$

where

$$
K\left(s_{m}, S_{m}, \theta_{2}\right)=\frac{1-S_{m}}{1-s_{m}}\left(1+\frac{\left(1-\theta_{2} / \delta\right)^{2}}{\left(1-\frac{\theta_{2}}{\delta}\right)\left(\frac{1-S_{m}}{\delta}\right)+\frac{\theta_{2}}{\delta^{2}}\left(\frac{\theta_{1}}{d}-s_{m}\right)}\right),
$$


and $\delta=S_{m}-s_{m}$. Here equation (??) is Bruno's lower bound for insulating inclusions in a matrix of unit conductivity. Collecting our results we display a lower bound on the effective conductivity of an imperfectly bonded composite that is a function of volume fraction, interfacial surface area, and the spectral parameters $s_{m}, S_{m}$. The bound is given by

$$
\sigma^{e} \geq \sigma_{1}-\sigma_{1}\left(\left(1-K\left(s_{m}, S_{m}, \sigma_{2}\right)\right)^{-1}+\left(\sigma_{1} \theta_{2} c\right)^{-1}\right)^{-1}
$$

Thus we are able to bound the effective transport properties for composites with imperfect interfaces in terms of the zeros and singularities of the effective conductivity function for composites with perfect contact between phases!

If the composite is made up of spherical inclusions then one can estimate $s_{m}$ and $S_{m}$ using the procedure of Bruno.

One also can appeal to the work of Torquato and Rubinstein (1991) to bound $m_{0}$ below for suspensions of spheres of diameter " $g$ ". Their lower bound is given by $m_{0} \geq J\left(\theta_{2}, H(x)\right)$ where

$$
J\left(\theta_{2}, H\right)=1+\theta_{2} g \int_{1}^{\infty} \frac{3}{2} x^{3} /\left(x^{3}-1\right) H(x) d x .
$$

Equation (??) is the lower bound of Torquato and Rubinstein and $H$ is the nearest neighbor distribution function for spherical inclusions of diameter $g$. The quantity $H(r) d r$ is the probability that given a sphere of diameter $g$ at the origin, the center of the nearest neighbor lies at a distance between $r$ and $r+d r$. In (??), $x=r / g$ represents dimensionless distance. Thus we are able to display an alternative lower bound on the effective conductivity for an imperfectly bonded composite that is determined by volume fraction, interfacial surface area, and nearest neighbor distribution function:

$$
\sigma^{e} \geq \sigma_{1}-\sigma_{1}\left(\left(1-J\left(\theta_{2}, H\right)\right)^{-1}+\left(\sigma_{1} \theta_{2} c\right)^{-1}\right)^{-1}
$$

We now consider the behavior of the bounds in the extreme cases $\beta=\infty$ and $\beta=0$. For $\beta=\infty$ the lower bound becomes:

$$
\sigma^{e} \geq \sigma_{1}-\sigma_{1}\left(\left(1-K\left(s_{m}, S_{m}, \theta_{2}\right)^{-1}-\frac{\sigma_{2}}{\theta_{2}\left(\sigma_{2}-\sigma_{1}\right)}\right)^{-1}\right.
$$

From the work of Bruno one has that the parameters $s_{m}$ and $S_{m}$ satisfy $s_{m} \leq \theta_{1} / d$ and $S_{m} \geq \theta_{1} / d+\theta_{2}$. For the choice, $s_{m}=\theta_{1} / d$ and $S_{m}=\theta_{1} / d+\theta_{2}$ the lower bound (??) reduces to the Hashin-Shtrikman lower bound for perfectly conducting isotropic composites in $d=2,3$. 
The case $\beta=0$ corresponds to a perfectly insulating interfacial surface. In this limit the bound (??) reduces to $\sigma_{1} m_{0}$. On the other hand one expects that this case is physically equivalent to a composite with insulating inclusions. Such composites have effective conductivity given precisely by $\sigma_{1} m_{0}$. Indeed, one observes that the objective function in the variational principle (I2.7) is monotone increasing in $1 / \beta$. Thus it follows from our earlier observations in I Section 4 that $\sigma^{e}=\sigma_{1} m_{0}$ for $\beta=0$. Therefore in the regime $\beta<<1$, the lower bound (??) provides a good estimate on the effective conductivity.

\section{II.2.2 Particles of low conductivity in a high conductivity matrix}

For the case of inclusions of conductivity $\sigma_{1}$ embedded in a matrix of conductivity $\left(\sigma_{2}>\sigma_{1}\right)$, we proceed as in the last section to obtain the lower bound for isotropic suspensions given by:

$$
\sigma^{e} \geq I C L_{21}\left(m_{0}, \beta\right)
$$

where,

$$
\begin{aligned}
& I C L_{21}\left(m_{0}, \beta\right)=\sigma_{1}+ \\
& +\quad \sigma_{1}\left[\frac{m_{0}\left[\frac{s}{d \beta}\left(1+\frac{\sigma_{2} \theta_{2}}{\sigma_{2}-\sigma_{1}}\right)-\theta_{2}^{2} \sigma_{1}^{-1}\right]-\frac{s}{d \beta} \frac{\sigma_{2} \theta_{2}}{\sigma_{2}-\sigma_{1}}+\theta_{2}^{2} \sigma_{1}^{-1}}{m_{0}\left[\left(\theta_{2}-\theta_{1}\right) \sigma_{1}^{-1}-\frac{s}{d \beta}-\frac{\sigma_{2} \theta_{2}}{\sigma_{1}\left(\sigma_{2}-\sigma_{1}\right)}\right]+\frac{s}{d \beta} \frac{\sigma_{2} \theta_{2}}{\sigma_{2}-\sigma_{1}}+\frac{\sigma_{2} \theta_{2}}{\sigma_{1}\left(\sigma_{2}-\sigma_{1}\right)}-\theta_{2}^{2} \sigma_{1}^{-1}}\right]
\end{aligned}
$$

For this geometry elementary Wiener bounds on $m_{0}$ give $0 \leq m_{0} \leq \theta_{2}$. Analysis shows that for $0 \leq m_{0} \leq \theta_{2}$ and $\beta>0$, one has $I C L_{21}\left(m_{0}, \beta\right)$ monotone increasing in $\beta$ and

$$
\sigma^{e} \geq I C L_{21}\left(m_{0}, \beta\right) \geq I C L_{21}\left(m_{0}, 0\right)=\sigma_{1}\left(\frac{1}{\frac{1}{m_{0}}-\frac{\sigma_{2}-\sigma_{1}}{\theta_{2} \sigma_{2}}}\right) \geq \sigma_{1} m_{0} .
$$

On the other hand $I C L_{21}\left(m_{0}, \beta\right)$ is found to be monotone increasing in $m_{0}$ for $\beta \geq 0$ and

$$
\sigma^{e} \geq I C L_{21}\left(m_{0}, \beta\right) \geq I C L_{21}(0, \beta)=\left(\frac{s}{d \beta}+\frac{\theta_{1}}{\sigma_{1}}+\frac{\theta_{2}}{\sigma_{2}}\right)^{-1} .
$$

In view of the monotonicity of $I C L_{21}\left(m_{0}, \beta\right)$ in $m_{0}$ we are able to use bounds on $m_{0}$ for composites with insulating inclusions to obtain bounds on $\sigma^{e}$. Indeed if 
we know the interval $\left[L_{1}, L_{2}\right]$ for the poles and zeroes of the effective conductivity function for perfect contact one has from (Bruno 1991)

$$
m_{0} \geq K\left(s_{m}, S_{m}, \theta_{1}\right)
$$

where $s_{m}=\frac{1}{1-L_{1}}$ and $S_{m}=\frac{1}{1-L_{2}}$ and $K$ is as given in (??). Substitution of the bound $K$ for $m_{0}$ in (??) yields a lower bound on the effective conductivity in terms of the spectral parameters $s_{m}, S_{m}$, the volume fraction, and the interfacial surface area.

For the case of mondisperse suspensions of spheres the work of Torquato and Rubinstein (1991) allows one to bound $m_{0}$ below by

$$
m_{0} \geq J\left(\theta_{1}, H(x)\right)
$$

Here $J$ is given by (??) and is in terms of the nearest neighbor distribution function. Substitution of $J$ for $m_{0}$ in (??) yields a lower bound on the effective conductivity in terms of nearest neighbor distribution function.

\section{II.3 New upper bounds on the effective conductivity}

Here we apply the upper variational principle (P2) of part I to obtain an upper bound on the effective conductivity. This bound incorporates partial geometric information on the composite geometry. In addition to volume fraction, the bound contains statistical information in terms of two point correlations and the moment of inertia tensors of the particle surfaces.

\section{II.3.1 Particles of high conductivity in a low conductivity matrix}

We consider particulate inclusions of conductivity $\sigma_{2}$ in a matrix of $\sigma_{1}$. We denote the region occupied by the $m^{\text {th }}$ particle by $Y_{m}$ and its boundary by $\partial Y_{m}$. To obtain the upper bound we make a suitable choice of bulk and surface polarization fields in the variational principle $(P 2)$ of part I. We choose polarizations of the form

$$
p(x)=\chi_{1} \mu \text { and } v(x)=r \cdot y^{m} \text { on } \partial Y_{m} .
$$

Here $\mu$ and $r$ can be any vectors in $\Re^{d}, \quad d=2,3$ and $y^{m}=x-r^{m}$ where $x$ lies on the surface of the $m^{t h}$ particle and $r^{m}$ is a reference point inside the particle. Upon 
substitution into the upper variational principle $(P 2)$ and choosing $\gamma=\sigma_{2}$, we obtain the upper bound:

$$
\left(\sigma^{e^{-1}}-\sigma_{2}^{-1}\right) \bar{\tau} \cdot \bar{\tau} \geq \max _{\mu \in \Re^{d}} \max _{r \in \Re^{d}}\{2 \bar{L}(\mu, r)-\bar{Q}(\mu, r)\}
$$

where,

$$
\begin{gathered}
\bar{L}(\mu, r)=\theta_{1} \mu \cdot \bar{\tau}+\sum_{m=1}^{N} \int_{\partial Y_{m}} n \otimes y^{m} d s r \cdot \bar{\tau} \\
\bar{Q}(\mu, r)=\theta_{1} \lambda|\mu|^{2}+\beta \sum_{m=1}^{N} \int_{\partial Y_{m}} y^{m} \otimes y^{m} d s \quad r \cdot r \\
+\sigma_{2}\left(\theta_{1} \theta_{2} \mathbf{I}-\mathbf{T}\right)(\mu-r) \cdot(\mu-r),
\end{gathered}
$$

Here

$$
\lambda=\left(\sigma_{1}^{-1}-\sigma_{2}^{-1}\right)^{-1} \text { and } \mathbf{T}=\int_{Q} \chi_{1} N \chi_{1} d x
$$

where $N$ is the projection onto periodic mean-zero curl-free fields introduced in (I2.26). We observe that the equation (??) for $\bar{L}$ and the first two terms in (??) follow directly upon substitution of the polarizations into $(P 2)$ of I. The last term appearing in (??) follows from substitution in the last term in (I 2.30). This substitution is nontrivial and requires proof. To facilitate the proof we require explicit formulas for the integral operators $N$ and $S$ defined in Part I, Section 2. We provide such formulas in the following Lemma.

Theorem II.3.1 The operator $N$ is the projection of any square integrable, periodic bulk polarization $p$ onto the space of mean zero gradients of periodic temperature fields and is defined locally in Fourier space by:

$$
N p=\sum_{k \neq 0} e^{2 \pi i k \cdot x} \frac{k \otimes k}{|k|^{2}} \hat{p}(k),
$$

The operator $S$ transforms square integrable fields $v$ on $\Gamma$ into gradients defined on the region $Y_{1} \cup Y_{2}$ and is represented locally in Fourier space by:

$$
S v=\sum_{k \neq 0} e^{2 \pi i k \cdot x}\left(\frac{k \otimes k}{|k|^{2}}-\mathbf{I}\right) \int_{\Gamma} e^{-2 \pi i k \cdot y} v n d s_{y}-\int_{\Gamma} v n d s_{y} .
$$

\section{PROOF.}

The explicit formulas and properties for the operator $N$ follows immediately from solution of the comparison problem (I2.20)-(I2.22) using Fourier expansions. This operator is well-known and forms the basis of the anisotropic Hashin Shtrikman bounds for composites with perfect interfaces given by Milton and Kohn (1988). 
To obtain the representation of the $S$ operator given by (??) we extend the function $v$ defined on $\Gamma$ into region-1. Where region-1 has a common boundary with the period cell we require the extension to be periodic there. It may be assumed that the extension of $v$ has a square integrable gradient in region-1. Denoting the extension of $v$ also by $v$ we introduce the auxiliary problem: for a periodic potential $w$ given by:

$$
\begin{gathered}
\Delta w=\operatorname{div}\left(\chi_{1} \nabla v\right) \text { in } Y_{1} \cup Y_{2}, \\
{\left[\nabla w-\chi_{1} \nabla v\right] \cdot n=0 \text { on } \Gamma}
\end{gathered}
$$

and

$$
[w]=0 \text { on } \Gamma
$$

It is evident from (??)-(??), that $\left(\nabla w-\chi_{1} \nabla v\right)$ is orthogonal to the subspace of all $Q$-periodic polarizations that can be written as gradients of periodic temperature fields, i.e.,

$$
\int_{Q}\left(\nabla w-\chi_{1} \nabla v\right) \cdot \nabla \delta d x=0
$$

Here $\delta$ is a $Q$-periodic vector field. Since $N$ is the projection onto the subspace of fields that can be written as gradients of potentials we have:

$$
N\left(\nabla w-\chi_{1} \nabla v\right)=0
$$

In addition from (??)-(??) it follows that, $N(\nabla w)=\nabla w$, thus we obtain:

$$
\nabla w=N\left(\chi_{1} \nabla v\right)
$$

From (??)-(??) we observe that the function defined by

$$
\begin{gathered}
\phi=w-v, \quad \text { in } Y_{1} \\
\phi=w, \quad \text { in } Y_{2}
\end{gathered}
$$

is a solution of the comparison problem (I2.23)-(I2.25). Since the solution $\phi^{v}$ of the comparison problem is unique up to a constant we may take $\phi^{v}$ to be represented by (??) and (??). It now follows from (??)-(??) that

$$
S v=\nabla \phi^{v}=\nabla w-\chi_{1} \nabla v \text { in } Y_{1} \cup Y_{2}
$$

Denoting the identity operator by $I$ we have from (??):

$$
S v=(N-I) \chi_{1} \nabla v \text { in } Y_{1} \cup Y_{2}
$$


In view of (??) equation (??) can be written as:

$$
S v=\sum_{k \neq 0}\left(e^{2 \pi i k \cdot x}\left(\frac{k \otimes k}{|k|^{2}}-\mathbf{I}\right) \widehat{\chi_{1} \nabla} v(k)\right)-\widehat{\chi_{1} \nabla v} v(0)
$$

From the divergence theorem we may write:

$$
\widehat{\chi_{1} \nabla} v(k)=\int_{\Gamma} e^{-2 \pi i k \cdot y} v n d s_{y}+(2 \pi i) k \int_{Q} e^{-2 \pi i k \cdot y} \chi_{i} v d y .
$$

Lastly noting that $\left((k \otimes k) /|k|^{2}-\mathbf{I}\right) k=0$ we obtain the desired representation $(? ?)$.

Next the formula for the last term in (??) follows from the:

Theorem II.3.2 For the choice $p=\chi_{1} \mu$ and $v=\tilde{v}(x)$ where $\tilde{v}=r \cdot y^{m}$ on $\partial Y_{m}$, the nonlocal term

$$
\int_{Q}\left|(N p+S v-p)-\int_{Q}(N p+S v-p) d x\right|^{2} d x
$$

in (I(2.30)) is given by:

$$
\left(\theta_{1} \theta_{2} \mathbf{I}-\mathbf{T}\right)(\mu-r) \cdot(\mu-r)
$$

Proof. Substitution of $p=\chi_{1} \mu$ and $v=\tilde{v}$ into (??) gives the expression:

$$
\begin{aligned}
\int_{Q} \chi_{1} N \chi_{1} d x \mu \cdot \mu & +\int_{Q}|S \tilde{v}|^{2} d x+\theta_{1}|\mu|^{2} \\
& -2\left[\int_{Q} \chi_{1} N \chi_{1} d x \mu \cdot \mu+\int_{Q} \chi_{1} S \tilde{v} d x \cdot \mu\right] \\
& -\left[\left|\int_{Q} S \tilde{v} d x\right|^{2}+\theta_{1}^{2}|\mu|^{2}-2 \theta_{1} \int_{Q} S \tilde{v} d x \cdot \mu\right]
\end{aligned}
$$

For the choice $v=\tilde{v}$ we appeal to Theorem 3.1 to find formulas for the terms $\int_{Q}|S \tilde{v}|^{2}, \int_{Q}(S \tilde{v}) \cdot \chi_{1} \mu d x$, and $\left|\int_{Q} S \tilde{v}\right|^{2}$. Indeed Theorem 3.1 equation (??) yields

$$
\begin{aligned}
S \tilde{v} & =\sum_{k \neq 0}\left[e^{2 \pi i k \cdot x}\left(\frac{k \otimes k}{|k|^{2}}-\mathbf{I}\right)\left(\sum_{m} \int_{\partial Y^{m}} e^{-2 \pi i k \cdot y}\left(r \cdot y^{m}\right) n d s\right)\right] \\
& -\sum_{m} \int_{\partial Y^{m}}\left(r \cdot y^{m}\right) n d s .
\end{aligned}
$$

We integrate by parts to find:

$$
\begin{aligned}
& \int_{\partial Y^{m}} e^{-2 \pi i k \cdot y}\left(r \cdot y^{m}\right) n d s \\
= & \left(\int_{Y_{m}} e^{-2 \pi i k \cdot y} d y\right) r-2 \pi i k \int_{Y_{m}} e^{-2 \pi i k \cdot y} r \cdot y^{m} d y .
\end{aligned}
$$


Noting that:

$$
\sum_{m} \int_{Y^{m}} e^{-2 \pi i k \cdot y} d y=\left\{\begin{array}{l}
\hat{\chi}_{2}(k)=-\hat{\chi}_{1}(k), \quad k \neq 0 \\
\theta_{2}, \quad k=0
\end{array}\right.
$$

and $\left(\frac{k \otimes k}{|k|^{2}}-\mathbf{I}\right) k=0$ it follows from (??), (??) and (??) that:

$$
S \tilde{v}=-\sum_{k \neq v} e^{2 \pi i k \cdot x} \hat{\chi}_{1}(k)\left(\frac{k \otimes k}{|k|^{2}}-\mathbf{I}\right) r-\theta_{2} r .
$$

Appealing to the explicit formula for the operator $N$ given in Theorem 3.1 it is evident from (??) that

$$
S \tilde{v}=(\mathcal{T}-N) \chi_{1} r-\theta_{2} r
$$

where $\mathcal{T}$ is the projection onto periodic square integrable mean-zero vector fields.

Observing that

$$
\int_{Q} \chi_{1} \mathcal{T} \chi_{1} r d x=\int_{Q} \chi_{1}\left(\chi_{1}-\theta_{1}\right) r d x=\theta_{1} \theta_{2} r
$$

it follows from (??) and (??) that

$$
\begin{gathered}
\int_{Q}|S \tilde{v}|^{2} d x=\left(\theta_{1} \theta_{2} \mathbf{I}-\mathbf{T}\right) r \cdot r+\theta_{2}^{2}|r|^{2}, \\
\int_{Q}(S \tilde{v}) \cdot \chi_{1} \mu d x=-\mathbf{T} r \cdot \mu,
\end{gathered}
$$

and

$$
\left|\int_{Q} S \tilde{v} d x\right|^{2}=-\theta_{2}|r|^{2}
$$

Formula (??) follows immediately upon substitution of (??) and (??) through (??) into (??).

The tensor $\mathbf{T}$ defined by (??) is well-known and appears in bounds on the effective conductivity for the perfect contact case, see Kohn and Milton(1988). This tensor $T$ contains two-point correlation information on the composite microstructure. Indeed $\mathbf{T}$ can be written as

$$
T_{i \ell}=\sum_{k \neq 0} \frac{k_{i} k_{\ell}}{|k|^{2}} \int_{Q} e^{2 \pi i k \cdot t} c_{b b}(t) d t,
$$

where $c_{b b}(t)$ is the two-point correlation:

$$
c_{b b}(t)=\int_{Q} \chi_{1}(x+t) \chi_{1}(x) d x .
$$


This function gives the probability that the ends of a rod of length and orientation described by the vector $t$ lies in both phases. This representation for $\mathbf{T}$ is easy to establish. Indeed from (??) and the formula for the operator $N$ given by (??) we can write

$$
T_{i \ell}=\sum_{k \neq 0} \frac{k_{i} k_{\ell}}{|k|^{2}}\left|\hat{\chi}_{1}(k)\right|^{2}
$$

Noting that $\left|\hat{\chi}_{1}(k)\right|^{2}=\chi_{1} \widehat{*} \chi_{1}(k)$ we see that $\left|\hat{\chi}_{1}(k)\right|^{2}$ is simply the Fourier transform of $c_{b b}(t)$ defined by (??). The relation between geometric tensors of the type given above and two-point correlations was observed in Willis (1982) and in the works of Avellaneda and Milton (1989).

We are now in a position to display new upper bounds on the effective conductivity for anisotropic composites in terms of second order geometric parameters. We introduce the tensors

$$
\mathbf{R}=\sum_{m} \int_{\partial Y^{m}} n \otimes y^{m} d s, \quad \mathbf{M}=\sum_{m} \int_{\partial Y^{m}} y^{m} \otimes y^{m} d s
$$

and carry out the optimization implied by (??) to obtain the upper bound: For any constant current field $\bar{\tau}$ one has,

$$
\left(\boldsymbol{\sigma}^{e^{-1}}-\sigma_{2}^{-1} \mathbf{I}\right) \bar{\tau} \cdot \bar{\tau} \geq\left(\begin{array}{cc}
\theta_{1} \lambda \mathbf{I}+\mathbf{A} & -\mathbf{A} \\
-\mathbf{A} & \beta \mathbf{M}+\mathbf{A}
\end{array}\right)^{-1}\left(\begin{array}{l}
\theta_{1} \bar{\tau} \\
\mathbf{R} \bar{\tau}
\end{array}\right) \cdot\left(\begin{array}{c}
\theta_{1} \bar{\tau} \\
\mathbf{R} \bar{\tau}
\end{array}\right)
$$

with $\mathbf{A}=\sigma_{2}\left(\theta_{1} \theta_{2} \mathbf{I}-\mathbf{T}\right)$.

We note that $\mathbf{M}=\sum_{m}\left(\frac{1}{2}\left(\operatorname{tr} \mathbf{J}_{m}\right) \mathbf{I}-\mathbf{J}_{m}\right)$ where $\mathbf{J}_{m}$ is the moment of inertia tensor for the surface of the mth particle. The two point correlation information enters the bound through the tensor $\mathbf{T}$. When the composite is statistically isotropic the tensor $\mathbf{T}$ is given by $\mathbf{T}=\frac{\theta_{1} \theta_{2}}{d} \mathbf{I} \quad(d=2,3)$, and the tensor $\mathbf{M}=d^{-1} \alpha \mathbf{I}$, where, $\alpha=\operatorname{tr} \mathbf{M}$. It follows from the relation

$$
\alpha=\operatorname{tr} \mathbf{M}=\sum_{m} \int_{\partial Y^{m}}\left|y^{m}\right|^{2} d s
$$

that the parameter $\alpha$ is equal to the sum of the polar moments of inertia of the surfaces $\partial Y^{m}$ with respect to the reference points $r^{m}$. For this case the bound given by (??) reduces to:

$$
\sigma^{e} \leq I C U_{12}(\alpha, \beta)
$$

where

$$
I C U_{12}(\alpha, \beta)=\left(\sigma_{2}^{-1}+\frac{\theta_{1} \beta d^{-1} \alpha+\theta_{2}^{2} \lambda+\theta_{2} \sigma_{2} d^{-1}(d-1)}{\lambda \beta d^{-1} \alpha+\theta_{1} \theta_{2} d^{-1}(d-1) \lambda \sigma_{2}+\theta_{2} d^{-2}(d-1) \sigma_{2} \beta \alpha}\right)^{-1}
$$


It is easily seen that $I C U_{12}$ is monotone increasing in the variables $\beta$ and $\alpha$. In view of this we may choose the reference points $r^{m}$ so as to minimize $\alpha$ to obtain the tightest bound. A straight forward calculation shows the best $r^{m}$ is given by

$$
r^{m}=\left(\left|\partial Y^{m}\right|\right)^{-1} \int_{\partial Y M} y d s,
$$

where $\left|\partial Y^{m}\right|$ is the surface area of the m-th particle. For perfectly bonded conductors $\beta=\infty$; passing to the $\beta=\infty$ limit in the upper bound (??) we recover the HashinShtrikman (1962) upper bound for isotropic two phase composites. Denoting the Hashin Shtrikman upper bound by $H S^{+}$we have from monotonicity

$$
I C U_{12}(\alpha, \beta) \leq H S^{+} \text {. }
$$

In the limit $\beta=0$ the upper bound becomes

$$
I C U_{12}(\alpha, 0)=\theta_{1} \sigma_{1}\left(\frac{1-d^{-1}}{1-\left(1-\theta_{2} \frac{\sigma_{1}}{\sigma_{2}}\right) d^{-1}}\right) .
$$

Noting that $\sigma_{2}>\sigma_{1}$ implies $\left(1-\theta_{2} \sigma_{1} / \sigma_{2}\right)>1-\theta_{2}=\theta_{1}$ we see that:

$$
I C U_{12}(\alpha, 0) \geq \theta_{1} \sigma_{1}\left(\frac{1-d^{-1}}{1-\theta_{1} d^{-1}}\right) \text {. }
$$

Here the right-hand side is the upper bound derived by Bruno (1991) for a composite with a matrix of $\sigma_{1}$ in the volume fraction $\theta_{1}$ filled with nonconducting material with volume fraction $\theta_{2}$.

\section{II.3.2 Particles of low conductivity in a high conductivity matrix}

For the case of inclusions of conductivity $\sigma_{1}$ embedded in a matrix of conductivity $\sigma_{2}$ we proceed as in the last section to obtain the upper bound for isotropic suspensions given by

$$
\sigma^{e} \leq I C U_{21}(\alpha, \beta)
$$

where

$$
I C U_{21}(\alpha, \beta)=\left(\sigma_{2}^{-1}+\frac{\theta_{1} \beta d^{-1} \alpha+\theta_{1}^{2} \lambda}{\lambda \beta d^{-1} \alpha+\theta_{1} \theta_{2} d^{-1}(d-1) \lambda \sigma_{2}+\theta_{2} d^{-2}(d-1) \sigma_{2} \beta \alpha}\right)^{-1}
$$

This bound is monotone increasing in the parameters $\alpha$ and $\beta$ and one has:

$$
\sigma_{2}\left(\frac{1-d^{-1}}{1-\theta_{2} d^{-1}}\right)=I C U_{21}(\alpha, 0) \leq I C U_{21}(\alpha, \beta) \leq I C U_{21}(\alpha, \infty)=H S^{+}
$$


The left hand side of the inequality (??) is precisely the upper bound derived by Bruno (1991) for a composite with a matrix of $\sigma_{2}$ in the volume fraction $\theta_{2}$ filled with nonconducting particles in the volume fraction $\theta_{1}$

\section{II.4 Concluding Remarks}

For isotropic particulate suspensions we have found new upper bounds depending upon the parameter $\alpha$, defined as the sum of polar moments of inertia of the particle surfaces with respect to their centroids given in (??) and volume fraction. Our new lower bounds depend upon surface area, volume fraction and the effective conductivity of a particulate composite with unit conductivity in the matrix phase and nonconducting particles having the same geometry as the imperfectly bonded composite.

The bounds presented in this paper were obtained from the new variational principles introduced in Part I. We observe that when trial fields of the type used in Section 3 are substituted into the basic variational principle (??) of Part I we obtain upper bounds that are not as tight as those given by $I C U_{12}(\alpha, \beta)$ derived in the last section (see equation (??)).

Indeed, for isotropic particulate suspensions of $\sigma_{2}$ in a matrix of $\sigma_{1}$ the best choice of trial fields is given by:

$$
\phi= \begin{cases}0 & \text { in the matrix } \\ \left(x-r^{m}\right) \cdot \eta & \text { in the } m^{t h} \text { particle }\end{cases}
$$

in (??) yields the upper bound $U_{12}(\alpha, \beta)$ :

$$
U_{12}(\alpha, \beta)=\theta_{1} \sigma_{1}+\theta_{2} \sigma_{2}\left(1-\frac{1}{1+\frac{\beta \alpha}{d \theta_{2} \sigma_{2}}}\right) .
$$

Direct calculation shows that $I C U_{12}$ is tighter than $U_{12}$, i.e.,

$$
\sigma^{e}(\beta) \leq I C U_{12}(\alpha, \beta) \leq U_{12}(\alpha, \beta)
$$

We summarize the principal results in the following inequalities: For isotropic particulate composites with matrix phase conductivity $\sigma_{1}$ and particle phase conductivity $\sigma_{2}$ we have,

$$
\sigma_{1} m_{0} \leq I C L_{12}\left(m_{0}, \beta\right) \leq \sigma^{e}(\beta) \leq I C U_{12}(\alpha, \beta) \leq U_{12}(\alpha, \beta)
$$




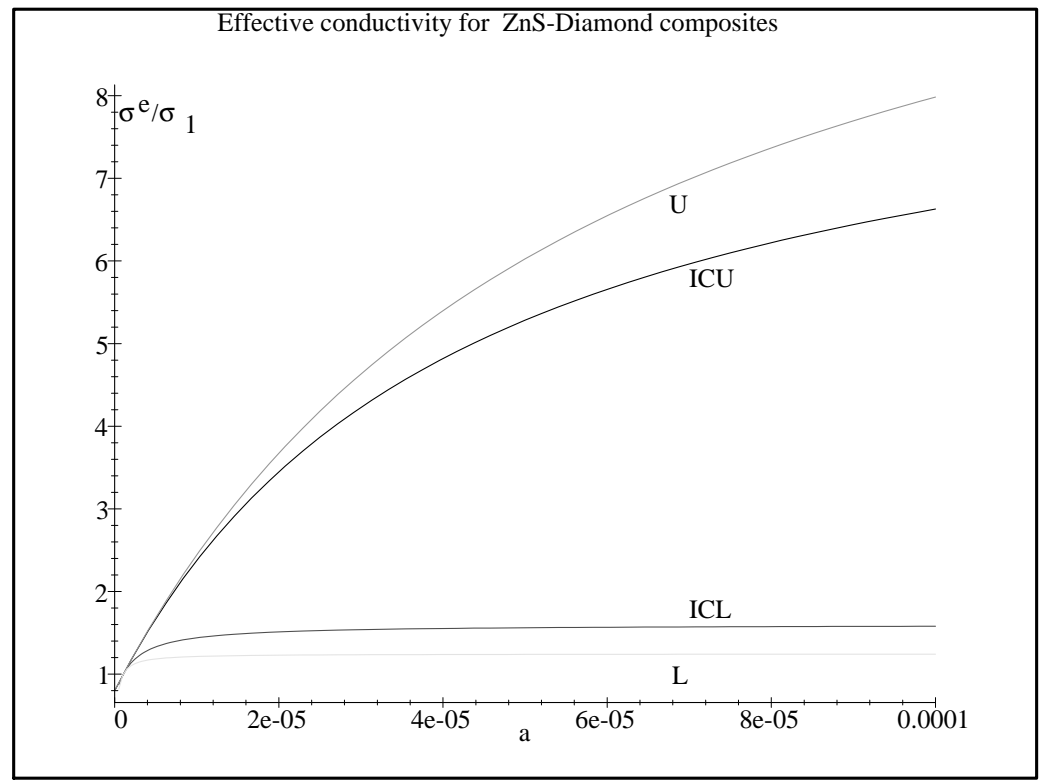

Figure 1: Interface comparison method upper bound ICU and lower bound ICL compared with the elementary upper bound (1.3) and the lower bound in (1.1) for periodic 2-d monodisperse suspensions of diamonds in a $\mathrm{ZnS}$ matrix with $\sigma_{1}=17.4 W /(m K), \sigma_{2}=1000 W /(m K), \theta_{2}=.2, \beta=6^{-1} 10^{8} W /\left(m^{2} K\right)$, and radii $a \leq 10 \mu m$. Here $s_{m}=\left(1-4 \pi / \theta_{2}\right) / 2, S_{m}=\left(1+4 \pi / \theta_{2}\right) / 2$ and the elementary upper and lower bounds are denoted by $\mathrm{U}$ and $\mathrm{L}$ respectively.

and

$$
\left(\frac{\theta_{1}}{\sigma_{1}}+\frac{\theta_{2}}{\sigma_{2}}+\frac{s}{d \beta}\right)^{-1} \leq I C L_{12}\left(m_{0}, \beta\right) \leq \sigma^{e}(\beta) \leq I C U_{12}(\alpha, \beta) \leq H S^{+} .
$$

When the phases are interchanged we have:

$$
\left(\frac{\theta_{1}}{\sigma_{1}}+\frac{\theta_{2}}{\sigma_{2}}+\frac{s}{d \beta}\right)^{-1} \leq I C L_{21}\left(m_{0}, \beta\right) \leq \sigma^{e}(\beta) \leq I C U_{21}(\alpha, \beta) \leq H S^{+} .
$$

To fix ideas we plot in Figure 1 the interface comparison upper bound (3.38) and lower bound (2.14) for periodic monodisperse suspensions of spheres. In the figure these upper and lower bounds are referred to as ICU and ICL, respectively.

In the next sections we analyze the behavior of the bounds $I C L_{12}, I C L_{21}$ at fixed volume fraction when the interfacial surface area is allowed to become infinite. We 
also present inequalities relating the geometric parameter $\alpha$ to interfacial surface area and analyze the behavior of $I C U_{12}, I C U_{21}$ in $\alpha$ for fixed volume fraction.

The analysis done in Part III yields new theoretical predictions of size effect phenomena for polydisperse suspensions of particles, (see Sections 6 and 7 of Part III).

\section{Size Effects and Extremal Microgeometries}

\section{III.1 Introduction}

In the previous sections we derived bounds on the effective conductivity for isotropic two phase particulate heat conductors with interfacial barrier resistance $\beta^{-1}$. These bounds depend upon component volume fractions $\theta_{1}, \theta_{2}$ interfacial surface area $s$, and the parameter $\alpha$ defined by:

$$
\alpha=\sum_{m} \int_{\partial Y^{m}}\left|y^{m}\right|^{2} d s
$$

where $\partial Y^{m}$ is the $m^{t h}$ particle boundary, $y^{m}=x-r^{m}, \quad r^{m}=\left|\partial Y^{m}\right|^{-1} \int_{\partial Y^{m}} y d s$, $\int_{\partial Y^{m}}\left|y^{m}\right|^{2} d s$ is the polar moment of inertia of the surface $\partial Y^{m}$, and $\alpha$ is the total polar moment of the inertia of interface. Here we display these bounds for particulate composites as functions of the geometric parameters. These bounds are compared with experimental results. For monodisperse suspensions of spheres of high conductivity $\sigma_{2}$ in a matrix of low conductivity $\sigma_{1}$ the parameter $\alpha$ is given by $d \theta_{2} r$, where $r$ is the sphere radius. For this case we exhibit a critical radius $R_{c r}=\beta^{-1} \sigma_{2} \sigma_{1}\left(\sigma_{2}-\sigma_{1}\right)^{-1}$, for which the effective conductivity equals that of the matrix. Our result is rigorous and follows from an exact analytical calculation of the temperature gradient in the composite. Using this result we are able to establish optimality of our bounds for certain values of interfacial surface area, total moment of inertia of the interface $\alpha$ and component volume fractions $\theta_{1}, \theta_{2}$.

We employ the upper bounds (II 3.38) to obtain new theoretical predictions of size effect phenomena for polydisperse suspensions of spherical conductors in a matrix of lesser conductivity. Here the spherical particles are of conductivity $\sigma_{2}$ and the matrix is of conductivity $\sigma_{1}$ with $\sigma_{2}>\sigma_{1}$. For this type of suspension the parameter $\alpha$ reduces to

$$
\alpha=d \theta_{2}\langle r\rangle
$$


where $\langle r\rangle$ is the "phase- 2 volume averaged" or mean radius given by

$$
\langle r\rangle=\sum_{m=1}^{N} \frac{\left|Y_{m}\right|}{\theta_{2}} r_{m},
$$

here $\left|Y_{m}\right|$ is the volume of the $m^{t h}$ sphere.

We apply our new upper bound (II3.38) to show that when the mean radius of the suspension lies below the critical value $R_{c r}$ the effective conductivity lies below that of the matrix, (see Theorem 6.1).

This prediction is rigorous and exact. Previous size effect predictions have been limited to monodisperse suspensions of spheres based upon approximate phenomenological models and low volume fraction expansions. Indeed, using the approach of Rayleigh and Maxwell, Hasselman and Johnson (1987) develop an effective medium theory. Using this model they are able to give the estimate $\sigma_{1} / \beta$ for the critical radius of a monodisperse suspension of spheres. Every et.al. (1992) also obtained the same estimate for a monodisperse suspension of spheres using a Differential Effective Medium Theory. It follows from our result (see Section 4,(??)) that their estimate is asymptotically correct in the limit $\sigma_{2}>\sigma_{1}$. For monodisperse dilute suspensions Chiew and Glandt (1987) show that $\sigma^{e}<\sigma_{1}$ for spherical particles of radius less than $R_{c r}$ and $\sigma^{e}>\sigma_{1}$ for particle radii greater than $R_{c r}$.

The result given in this paper is, to the best of our knowledge the first theoretical size effect prediction for polydisperse suspensions of spheres at high concentration.

In Section 7 we investigate the general question of optimal design of isotropic particulate suspensions of conducting particles in a matrix of lesser conductivity at fixed volume fraction. In this context we apply the monotonicity of the lower bound (II 1.1) to show that the effective conductivity is greater than the matrix provided the interfacial surface area is less than the value $d \theta_{2} R_{c r}^{-1}$ see (Theorem 7.1).

Next we consider the problem of the optimal isotropic distribution of conducting spheres of different radii in a matrix of lesser conductivity. For fixed volume fraction of spherical particles, the objective is to find the optimal distribution of spheres that give the best effective heat transport properties in all directions, (i.e., maximize the effective conductivity). We employ Theorem 6.1 and Theorem 7.1 to show that any polydisperse distribution with mean radius less than $R_{c r}$ is suboptimal. Thus we see that the scale of the heterogeneties has an effect on the optimality of a particular design. This is in striking contrast to optimal layout problems with perfect heat transmission between phases where scale plays no role in the optimal design, see 
Lurie and Cherkaev (1986), Murat and Tartar (1985).

For isotropic monodisperse suspensions of spheres we can say more. Indeed Theorems 6.1 and 7.1 imply that:

1. For spheres of radius less than $R_{c r}$ the effective property lies below that of the matrix.

2. For spheres of radius greater than $R_{c r}$ the effective property lies strictly above that of the matrix (see Theorem 7.3).

These results constitute the first rigorous proof of size effect phenomena for monodisperse suspensions at high volume fraction.

We may also apply the monotonicity of the upper and lower bounds in the geometric parameters, $s$ and $\alpha$ to solve inverse problems. Indeed, for an isotropic particulate suspension of isotropic conductors in a matrix of lesser conductivity we show that: when the effective conductivity is greater than that of the matrix then the total moment of inertia of the interface is greater than $d \theta_{2} R_{c r}$, (see Theorem 8.1). For the

special case of polydisperse suspensions of spheres one has a similar result in terms of the mean radius, (see Corollary 8.1). We find that when the effective property is less than the matrix the ratio of particle volume to interfacial surface $\theta_{2} / s$ is less than $R_{c r}$, (see Theorem 8.2).

Lastly we use monotonicity of the bounds in $\beta$ to recover estimates for interface resistivity from measured values of the effective conductivity, see Theorem 8.3.

\section{III.2 Inequalities between geometric parameters}

The bounds derived in the previous article (Part II) were in terms of the geometric parameters, $s, \alpha$, and the component volume fractions $\theta_{1}, \theta_{2}\left(\theta_{1}+\theta_{2}=1\right)$. For isotropic suspension of particles of conductivity $\sigma_{2}$ in a matrix of conductivity $\sigma_{1}$ the parameters satisfy the following inequality:

$$
d^{2} \theta_{2}\left(\frac{\theta_{2}}{s}\right) \leq \alpha
$$

Inequality (??) follows from two applications of Cauchy's inequality. To see this we derive the preliminary inequality:

$$
d^{2}\left(\sum_{m}\left|Y^{m}\right|\left(\frac{\left|Y^{m}\right|}{\left|\partial Y^{m}\right|}\right)\right) \leq \alpha .
$$


Inequality (??) follows immediately from the definition of $\alpha$ and the term by term estimate,

$$
\left(\int_{\partial Y^{m}} y^{m} \cdot n d s\right)^{2} \leq\left|\partial Y^{m}\right| \int_{\partial Y^{m}}\left|y^{m}\right|^{2} d s
$$

where $n$ is the unit outward directed normal to the $m^{\text {th }}$ particle surface $\partial Y^{m}$. Integration by parts on the left hand side of (??) yields:

$$
d^{2}\left|Y^{m}\right|^{2} \leq\left|\partial Y^{m}\right| \int_{\partial Y^{m}}\left|y^{m}\right|^{2} d s
$$

and (??) follows. We apply Cauchy's inequality to obtain:

$$
d^{2} \frac{\left(\sum\left|Y^{m}\right|\right)^{2}}{\sum\left|\partial Y^{m}\right|} \leq d^{2}\left(\sum_{m}\left|Y^{m}\right| \frac{\left|Y^{m}\right|}{\left|\partial Y^{m}\right|}\right),
$$

Noting that $\theta_{2}=\sum\left|Y^{m}\right|$ and $s=\sum\left|\partial Y^{m}\right|$ inequality (??) follows immediately from (??).

\section{III.3 Asymptotic behavior of the bounds with respect to geometric parameters}

We consider the behavior of the lower bound as we increase interfacial surface while keeping the particulate volume fraction fixed. We also study the upper bound when we allow the total polar moment of inertia of the interface " $\alpha$ ", to decrease to zero. It follows from (??) that the limit $\alpha \rightarrow 0$ implies that interfacial surface area tends to infinity. It is interesting to note that all bounds in these limits reduce to bounds on the effective conductivity associated with composites formed from nonconducting inclusions.

\section{III.3.1 Particles of high-conductivity in a low conductivity matrix}

For isotropic particulate composites with particles of $\sigma_{2}$ in a matrix of lesser conductivity $\sigma_{1}$ we have the lower bound as given by equation (II 2.10). We fix volume fractions and pass to the infinite interfacial surface area limit to find that the lower bound becomes exactly $\sigma_{1} m_{0}$ where $\sigma_{1} m_{0}$ is the conductivity associated with the same microgeometry but with nonconducting particles and a matrix of conductivity $\sigma_{1}$. 
The upper bound for such composites at fixed volume fraction depends upon the $\alpha$ parameter and is given by (II 3.38). Passing to the $\alpha=0$ limit the bound becomes:

$$
\theta_{1} \sigma_{1}\left(\frac{1-d^{-1}}{1-\left(1-\theta_{2} \frac{\sigma_{1}}{\sigma_{2}}\right) d^{-1}}\right)
$$

Since $\left(1-\theta_{2} \sigma_{1} / \sigma_{2}\right)>1-\theta_{2}=\theta_{1}$ it follows that the above limit is larger than:

$$
\theta_{1} \sigma_{1}\left(\frac{1-d^{-1}}{1-\theta_{1} d^{-1}}\right) \text {. }
$$

Here formula (??) is precisely the upper bound on composites of matrix conductivity $\sigma_{1}$ with insulating inclusions given by Bruno (1991).

\section{III.3.2 Particles of low conductivity in a high conducting matrix}

In the case of particulate composites with particles of conductivity $\sigma_{1}$ in a matrix of higher conductivity $\sigma_{2}$. The lower bound is given by (II 2.19). By fixing the volume fractions and considering the limit as the interfacial surface area tends to infinity, the lower bound becomes:

$$
\sigma_{2} m_{0}\left(\frac{m_{0}}{\theta_{2}}+\left(1-\frac{m_{0}}{\theta_{2}}\right) \frac{\sigma_{2}}{\sigma_{1}}\right)^{-1}
$$

Since $m_{0}<\theta_{2}$ the convex combination above lies between 1 and $\frac{\sigma_{2}}{\sigma_{1}}>1$. Therefore the limit in (??) is less than $\sigma_{2} m_{0}$, which represents the effective conductivity of a composite with the same microgeometry but with nonconducting particles in a $\sigma_{2}$ conductivity matrix.

The upper bound for this case is given in (II 3.44). When $\alpha$ tends to zero the bound becomes:

$$
\sigma_{2} \theta_{2}\left(\frac{1-d^{-1}}{1-\theta_{2} d^{-1}}\right)
$$

which represents the upper bound for composites of conductivity $\sigma_{2}$ with insulating inclusions (see Bruno (1991)).

\section{III.4 Effective behavior for monodisperse suspensions of spheres at critical particle size}

It is known from experiment that particle size affects the overall thermal transport properties of composites, see (c.f., Garret and Rosenberg (1974), Every et. al. (1992)). 
In this section we consider a monodisperse suspension of spheres of good conductor $\sigma_{2}$ embedded in a matrix of conductivity $\sigma_{1}$. For prescribed barrier resistance $\beta^{-1}$, we exhibit a critical particle radius $R_{c r}$ for which the effective transport properties are identical to the conductivity of the matrix material. At this critical size the effect of the interface resistance is balanced by the higher conductivity of the particles. Denoting the critical radius by $R_{c r}$ we have:

$$
R_{c r}=\beta^{-1} \sigma_{2} \sigma_{1}\left(\sigma_{2}-\sigma_{1}\right)^{-1}
$$

We begin with the obvious remark that for composites occupying the unit cube we only consider parameter values $\beta, \sigma_{2}, \sigma_{1}$ for which the right hand side of (??) is less than $1 / 2$.

To establish our formula for the critical radius we consider a dispersion of $N$ spheres of common radius " $a$ " with centers denoted by the vectors $r^{i}$. We assume all spheres are contained in the unit cube and do not touch. We show that for $a=R_{c r}$ there exists a periodic piecewise affine solution to the problem (I2.2)-(I2.3) of the form:

$$
\phi+\xi \cdot x= \begin{cases}\xi \cdot x & \text { in the matrix } \\ \frac{\sigma_{1}}{\sigma_{2}} \xi \cdot x-\left(1-\frac{\sigma_{2}}{\sigma_{1}}\right) \frac{\sigma_{1}}{\sigma_{2}} \xi \cdot r^{i}, & \text { in the ith particle }\end{cases}
$$

For this choice of temperature field the effective conductivity given by (I2.5) reduces to $\sigma^{e}=\sigma_{1}$.

We look for a solution of the form:

$$
\phi+\xi \cdot x= \begin{cases}\xi_{A} \cdot x & \text { in the matrix } \\ \xi_{B} \cdot x+c_{i} & \text { in the ith particle. }\end{cases}
$$

From (I2.1), (I2.3), and (I2.4) it follows that:

$$
\begin{aligned}
\xi+\int_{\Gamma}[\phi] n d s & =\theta_{1} \xi_{A}+\theta_{2} \xi_{B}, \\
\sigma_{1} \xi_{A} \cdot n & =\sigma_{2} \xi_{B} \cdot n
\end{aligned}
$$

and

$$
\sigma_{2} \xi_{B} \cdot n=-\beta\left\{\left(\xi_{B}-\xi_{A}\right) \cdot x+c_{i}\right\} .
$$

From (??) we may conclude that $\frac{\sigma_{2}}{\sigma_{1}} \xi_{B}=\xi_{A}$. On the surface of the ith sphere the unit normal is written $n=\left(x-r_{i}\right) / a$, thus $x=a n+r^{i}$ on the surface and (??) can be written as

$$
\xi_{B} \cdot n\left(\sigma_{2}+\beta a\left(1-\frac{\sigma_{2}}{\sigma_{1}}\right)\right)=-\beta\left(\left(1-\frac{\sigma_{2}}{\sigma_{1}}\right) \xi_{B} \cdot r^{i}+c_{i}\right)
$$


It is seen from (??) that both sides must equal zero thus:

$$
\begin{gathered}
\xi_{B}\left(\sigma_{2}+\beta a\left(1-\frac{\sigma_{2}}{\sigma_{1}}\right)\right)=0 \\
\left(1-\frac{\sigma_{2}}{\sigma_{1}}\right) \xi_{B} \cdot r^{i}+c_{i}=0 .
\end{gathered}
$$

We observe from (I2.4) that

$$
[\phi]=-\frac{\sigma_{2}}{\beta} \xi_{B} \cdot n=-\frac{\sigma_{2}}{\beta} \xi_{B} \cdot \frac{x-r^{i}}{a}
$$

and (??) becomes:

$$
\xi_{B}=\left(\theta_{1} \frac{\sigma_{2}}{\sigma_{1}}+\theta_{2}+\frac{\theta_{2} \sigma_{2}}{\beta a}\right)^{-1} \xi .
$$

It is evident from (??) that either

$$
\xi_{B}=0
$$

or,

$$
\sigma_{2}+\beta a\left(1-\frac{\sigma_{2}}{\sigma_{1}}\right)=0
$$

For finite values of $\beta, \sigma_{2}, \sigma_{1}$ we may rule out the case $\xi_{B}=0$ as this implies $\xi=0$ from (??).

Equation (??) provides the relation defining the critical radius $R_{c r}$ and is equivalent to (??). For the choice of "a" given by (??) we find:

$$
\xi_{B}=\frac{\sigma_{1}}{\sigma_{2}} \xi
$$

Last we deduce from (I2.5) that

$$
\sigma^{e} \xi=\theta_{1} \sigma_{1} \xi_{A}+\theta_{2} \sigma_{2} \xi_{B}
$$

and from the relation $\xi_{A}=\frac{\sigma_{2}}{\sigma_{1}} \xi_{B}$ and (??) it follows that $\sigma^{e} \xi=\sigma_{1} \xi$ for any choice of $\xi$. Formula (??) follows from (??) and the above remarks.

The above analysis shows that at the critical radius the overall heat conductance remains that of the matrix irrespective of the location and number of particles.

We point out that the Differential Effective Medium Model introduced by Every et.al. (1992) predicts that $\sigma^{e}=\sigma_{1}$ for spherical particles with radius $\sigma_{1} / \beta$. We remark that in light of our exact calculation their result provides only an estimate of the critical radius. However for large values of the particle conductivity their estimate approaches the exact value given by (??). 


\section{III.5 Critical values of the geometric parameters and opti- mality}

We examine the behavior of the bounds (II2.10) and (II3.38) in the parameters $\alpha$ and $s$ for suspensions of particles with conductivity $\sigma_{2}$ in a matrix of $\sigma_{1}$ with $\sigma_{2}>\sigma_{1}$. Indeed, when the total surface moment of inertia $\alpha=\theta_{2} d R_{c r}$ the upper bound given by (II3.38) reduces to:

$$
I C U_{12}\left(\theta_{2} d R_{c r}, \beta\right)=\sigma_{1} .
$$

Similarly when the surface area $s=d \theta_{2} R_{c r}^{-1}$ then the lower bound given by (II2.10) becomes:

$$
I C L_{12}\left(m_{0}, \beta\right)=\sigma_{1}
$$

Now we show for the choice $\alpha=\theta_{2} d R_{c r}$ and $s=d \theta_{2} R_{c r}^{-1}$ that the upper and lower bounds are optimal. We observe from the results of previous section that for a monodisperse suspension of spheres of critical radius " $R_{c r}$ " of conductivity $\sigma_{2}$ in a matrix of $\sigma_{1}$ that:

$$
\sigma^{e}=\sigma_{1}
$$

Moreover, for this suspension the associated geometric parameters $\alpha$ and $s$ are given by:

$$
\alpha=\theta_{2} d R_{c r}, \quad s=\theta_{2} d R_{c r}^{-1}
$$

Thus the bounds (??) and (??) are simultaneously obtained by a monodisperse suspension of spheres of critical radius $R_{c r}$. To fix ideas we plot in Figure 1 the upper and lower bounds (II(3.38)) and (II2.14)) for monodisperse periodic suspensions in the $d=2$ case. We also compare these to the simpler bounds given by (II1.3) and (II1.1).

\section{III.6 Size effect phenomena for polydisperse suspensions of spheres}

For polydisperse suspensions of spheres the parameter $\alpha=d \theta_{2}\langle r\rangle$, (c.f. (??)) where $\langle r\rangle$ is the mean radius of the spherical inclusions given by (??) . For spheres of $\sigma_{2}$ in a matrix of $\sigma_{1}$ the upper bound (II3.38) becomes:

$$
I C U_{12}\left(d \theta_{2}\langle r\rangle, \beta\right)=\left(\sigma_{1}^{-1}+\frac{\theta_{1} \theta_{2} \beta\langle r\rangle+\theta_{2}^{2} \lambda+\theta_{2} \sigma_{2} d^{-1}(d-1)}{\theta_{2} \lambda \beta d^{-1}\langle r\rangle+\theta_{1} \theta_{2} d^{-1}(d-1) \lambda \sigma_{2}+\theta_{2}^{2} d(d-1) \sigma_{2} \beta\langle r\rangle}\right)^{-1}
$$




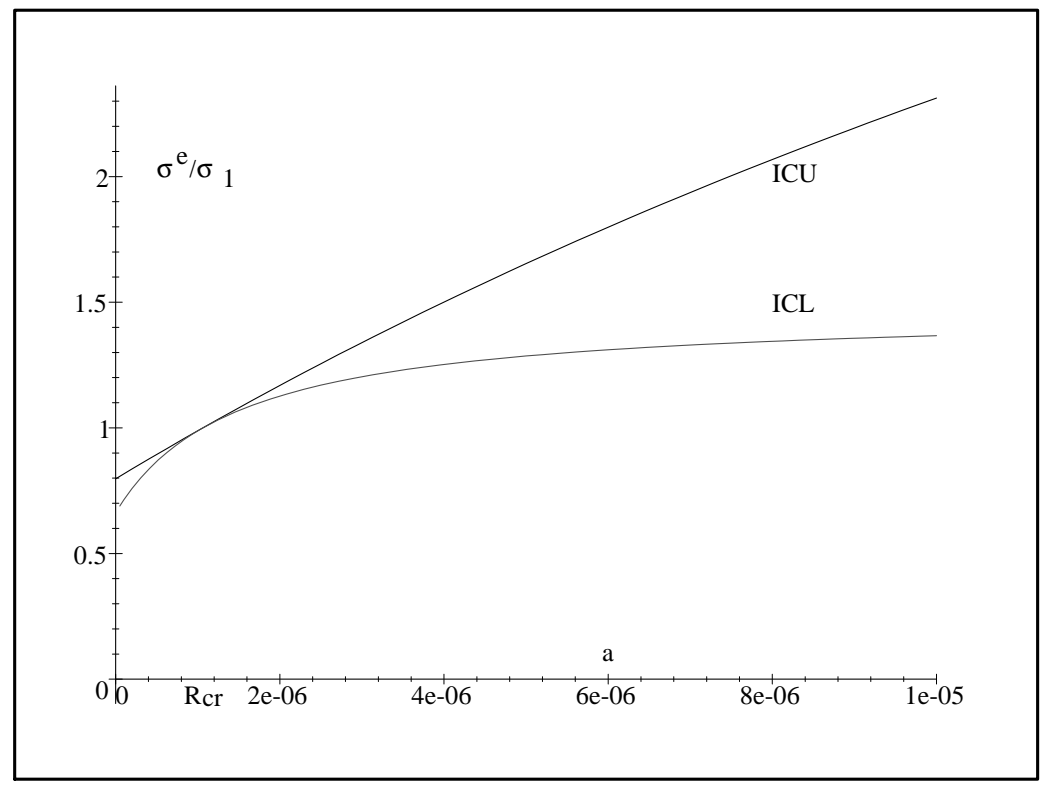

Figure 2: Interface comparison method upper and lower bounds for periodic 2-d monodisperse suspensions of diamonds in a $\mathrm{ZnS}$ matrix with $\sigma_{1}=17.4 \mathrm{~W} /(\mathrm{mK}), \sigma_{2}=$ $1000 W /(m K), \theta_{2}=.2, \beta=6^{-1} 10^{8} W /\left(m^{2} K\right)$, and radii $a \leq 10 \mu m$ (here $s_{m}=(1-$ $\left.\left.4 \pi / \theta_{2}\right) / 2, S_{m}=\left(1+4 \pi / \theta_{2}\right) / 2\right)$.

where $\lambda=\sigma_{1} \sigma_{2}\left(\sigma_{2}-\sigma_{1}\right)^{-1}$.

This bound is strictly monotone increasing in $\langle r\rangle$ and for $\langle r\rangle=R_{c r}$

$$
I C U_{12}\left(d \theta_{2} R_{c r}, \beta\right)=\sigma_{1}
$$

From monotonicity and (??) we have the:

Theorem III.6.1 (Size Effect Theorem): For polydisperse suspensions of spheres of $\sigma_{2}$ in a matrix $\sigma_{1}$ with $\sigma_{2}>\sigma_{1}$ and any prescribed volume fraction $\theta_{2}$, if $\langle r\rangle \leq R_{c r}$ then:

$$
\sigma^{e} \leq \sigma_{1}
$$

One has equality in (??) only if:

$$
\langle r\rangle=R_{c r}
$$




\section{III.7 Necessary conditions of optimal design}

We consider suspensions of a $\sigma_{2}$ particles in a matrix of $\sigma_{1}$ with volume fractions $\theta_{1}, \theta_{2}$ of the constituent materials fixed. We recall the lower bound (II 1.1) is given by:

$$
\begin{gathered}
\sigma^{e} \geq\left(\frac{\theta_{1}}{\sigma_{1}}+\frac{\theta_{2}}{\sigma_{2}}+\frac{s}{d \beta}\right)^{-1}= \\
=\sigma_{1}\left(1-\frac{\sigma_{1} \theta_{2} \beta^{-1}\left(\frac{s}{d \theta_{2}}-R_{c r}^{-1}\right)}{1+\sigma_{1} \theta_{2} \beta^{-1}\left(\frac{s}{d \theta_{2}}-R_{c r}^{-1}\right)}\right) .
\end{gathered}
$$

From (??) we see the lower bound equals $\sigma_{1}$ for $s=d \theta_{2} R_{c r}^{-1}$. It is evident from (??) that the lower bound is strictly monotone decreasing in the total interfacial surface area $s$. We collect these observations and state the following theorem:

Theorem III.7.1 For an isotropic suspension of $\sigma_{2}$ conducting particles in a matrix of $\sigma_{1}$ conductivity with $\sigma_{2}>\sigma_{1}$ and any prescribed volume fraction $\theta_{2}$, if the total interfacial surface is bounded above by $d \theta_{2} R_{c r}^{-1}$ then:

$$
\sigma^{e}>\sigma_{1}
$$

We now consider the problem of the best isotropic distribution of good and bad conductors in the unit cell $Q$. Here the best distribution is the one giving the best effective heat transport in all directions, (i.e., the largest value of the effective conductivity). It is assumed that the volume fraction of good conductor $\sigma_{2}$ is fixed at $\theta_{2}$.

In what follows we investigate the effect of scale in problems of optimal design. To fix ideas we suppose that the volume fraction of the good conductor satisfies the inequality:

$$
\theta_{2}<\frac{2(d-1)}{d} \pi 2^{-d}, \quad d=2,3 .
$$

That is the volume fraction is less than that of a sphere (circle) of radius $1 / 2$ inscribed within the unit cell. Moreover we restrict the parameters $\beta, \sigma_{1}, \sigma_{2}$ so that the critical radius $R_{c r}$ satisfies the constraint:

$$
\frac{2(d-1)}{d} \pi R_{c r}^{d}<\theta_{2}, \quad d=2,3 .
$$

The above states that we consider only cases where the volume of a single sphere of critical radius is strictly less than the volume fraction $\theta_{2}$ occupied by the polydisperse suspension. 
We consider the best distribution of an isotropic, polydisperse, suspension of spheres of good conductor $\sigma_{2}$ in a matrix of $\sigma_{1}$, for $\theta_{2}$ prescribed and the given constraints (??) and (??). We have the following theorem characterizing the optimal design:

\section{Theorem III.7.2 (Optimal Design-Necessary Condition)}

The mean radius of the optimal distribution of spheres maximizing the effective conductivity is greater than $R_{c r}$.

Proof. From Theorem 6.1 we know that if the mean radius lies below $R_{c r}$ then $\sigma^{e} \leq \sigma_{1}$. So to establish the theorem we construct a polydisperse suspension of spheres with mean radius greater than $R_{c r}$ such that the effective conductivity lies above $\sigma_{1}$. The construction is trivial in view of (??) and (??). Indeed take a suspension consisting of a single sphere of radius $r$ such that:

$$
\frac{2(d-1)}{d} \pi r^{d}=\theta_{2}
$$

Then for this suspension

$$
\frac{s}{d \theta_{2}}=\frac{1}{r}
$$

From (??) and (??) we see that

$$
r^{-1}<R_{c r}^{-1}
$$

therefore

$$
s<d \theta_{2} R_{c r}^{-1}
$$

We conclude from (??) and Theorem 7.1 that the effective conductivity for this suspension lies above $\sigma_{1}$ and the Theorem is established.

We conclude this section by making an observation for isotropic monodisperse suspensions of spheres of radius $r$. For this case $\alpha=d \theta_{2} r$ and $s=d \theta_{2} r^{-1}$. Thus we may apply Theorems 6.1 and 7.1 to obtain:

Theorem III.7.3 For isotropic monodisperse suspensions of spheres of radius $r$ at prescribed volume fraction:

$$
\sigma^{e}>\sigma_{1} \text { if } r>R_{c r}
$$

and

$$
\sigma^{e}<\sigma_{1} \text { if } r<R_{c r}
$$


Such a behavior is illustrated for ZnS-Diamond composites in Figure 1.

We consider the effect of varying the volume fraction at a fixed particle size for isotropic composites. The bounds on the effective conductivity given by (II3.38) and (II2.14) are plotted in Figure 2 for a periodic suspension of Diamonds in a ZnS matrix. We observe that the upper and lower bounds are increasing functions in volume fraction for a particle of size $2 \mu \mathrm{m}$, larger than $R_{c r}=1.06 \mu \mathrm{m}$, and that the bounds are decreasing functions in volume fraction for a particle size of $.25 \mu \mathrm{m}$. For $a=R_{c r}=1.06 \mu \mathrm{m}$ the upper and lower bound are both equal to $\sigma_{1}$. These bounds predict the same behavior seen in the experimental results of Every et. al. (1992).

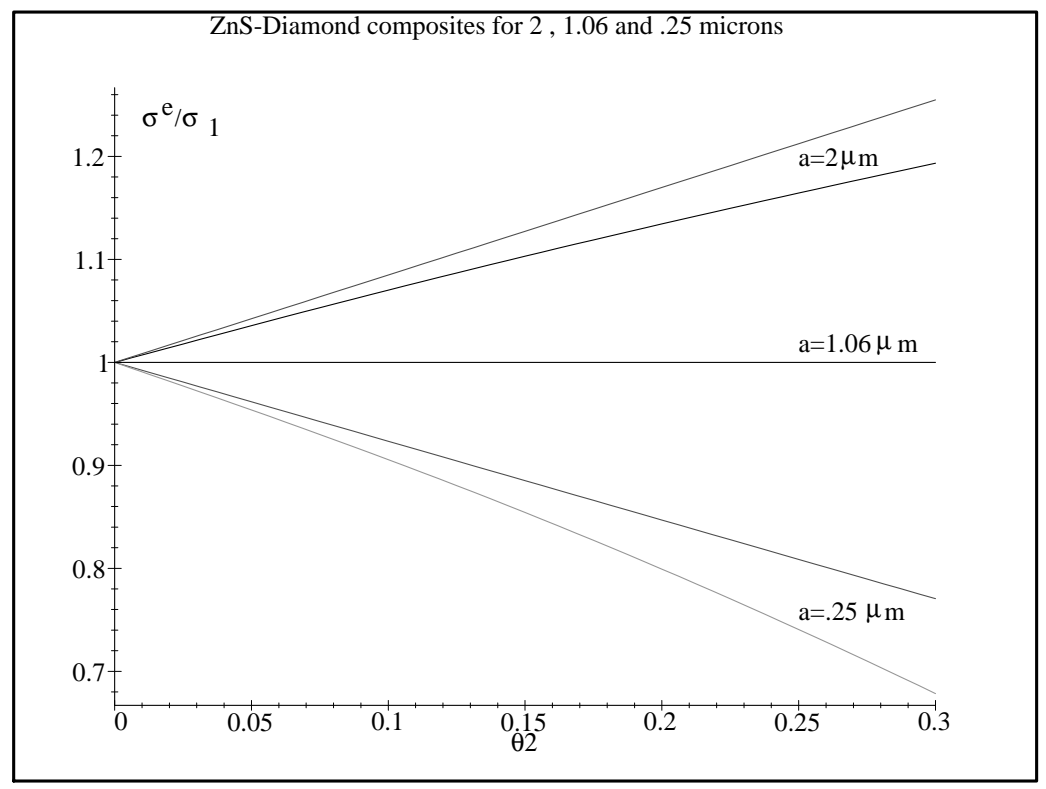

Figure 3: Interface comparison method upper and lower bounds for ZnS-Diamond composites as a function of the volume fraction of the diamond, for different diamond particle sizes: $a=2 . \mu m, a=R_{c r}=1.06 \mu m, a=.25 \mu m$.

Lastly we plot the interface comparison method bounds given by (II3.38) and (II2.14) for a periodic suspension of diamonds in a matrix of $\mathrm{ZnS}$ as a function of particle radius and volume fraction in Figure 3. 


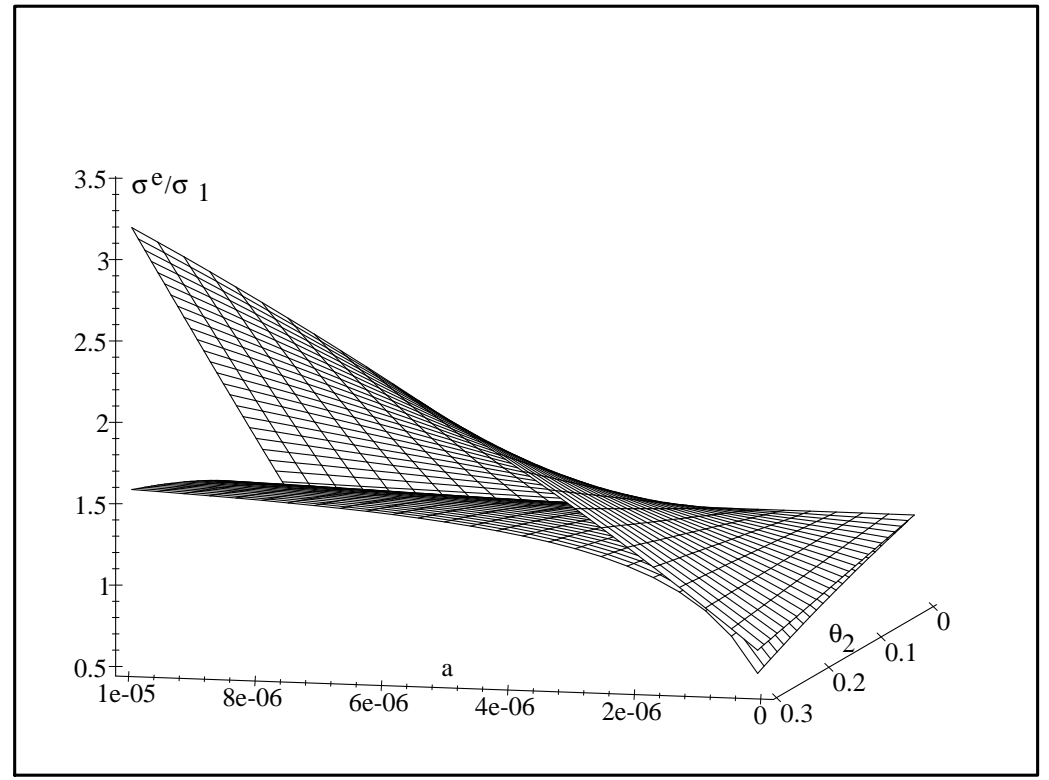

Figure 4: Interface comparison method upper and lower bounds for monodisperse suspensions of diamond in a $\mathrm{ZnS}$ matrix as functions of volume fraction $\theta_{2}$ and particle radius $a$.

\section{III.8 Inverse Problems}

The upper and lower bounds (II2.10) and (II3.38) are monotonic in the parameters $s, \alpha$ and $\beta$. We use this property to gather information on the composite geometry from measured values of the effective conductivity. We limit the scope of our discussion to isotropic particulate composites of conductors in a matrix of lesser conductivity, i.e., particles of conductivity $\sigma_{2}$ in a matrix of $\sigma_{1}$ with $\sigma_{2}>\sigma_{1}$.

We shall assume that the volume fractions $\theta_{2}$ and $\theta_{1}$ are known as well as the values $\sigma_{2}, \sigma_{1}$ and $\beta$ and bound the geometric parameters $s$ and $\alpha$ from measured values of the effective conductivity $\sigma^{e}$. The upper bound (II3.38) is easily seen to be monotonically increasing in $\alpha$, thus from (??) we have the following:

Theorem III.8.1 If $\sigma^{e}>\sigma_{1}$ then the total moment of inertia of the interface " $\alpha$ " satisfies:

$$
\alpha>d \theta_{2} R_{c r}
$$

For isotropic polydisperse suspensions with mean radius $\langle r\rangle$ as defined by (??) we 
have the:

Corollary III.8.1 If $\sigma^{e}>\sigma_{1}$ then $\langle r\rangle>R_{c r}$.

On the other hand the lower bound is monotonically decreasing in the interfacial surface area thus from (??) it follows that:

Theorem III.8.2 If $\sigma^{e}<\sigma_{1}$ then the particle volume to interfacial surface area ratio satisfies:

$$
\theta_{2} / s<R_{c r}
$$

Using monotonicity of the bounds in the parameter $\beta$ for fixed component volume fraction and surface area, we obtain bounds on the surface conductivity in terms of measured values of the effective property.

Theorem III.8.3 For given values of the geometric parameters $\alpha, s, \theta_{2}$ and for given effective conductivity $\sigma^{e}$

$$
\underline{\beta}<\beta<\bar{\beta}
$$

where $\underline{\beta}$ and $\bar{\beta}$ are the unique solutions of:

$$
I C U_{12}(\alpha, \underline{\beta})=\sigma^{e}, I C L_{12}\left(m_{0}, \bar{\beta}\right)=\sigma^{e} .
$$

R. Lipton supported by NSF grant DMS-9205158.

\section{REFERENCES}

Avellaneda, M. and Milton, G. W. 1989 Bounds on the effective elasticity of composites based on two-point correlations. In Proc. 5th Energy - Technology Conf. Exhibition: Composite Materials (eds. D. Hui and T. Kozic). ASME, New York.

Attouch, H. and Wets, R. 1983 A convergence theory for saddle functions. Trans. A.M.S. 280, 1-41

Benveniste, Y. 1986 Effective thermal conductivity of composites with a thermal contact resistance between the constituents: Nondilute case. J.Appl.Phys. 61 $2840-2844$ 
Bergman, D. J. 1978 The dielectric constant of a composite material - A problem in classical physics. Physics Report 43, 378-407.

Bhatt, H., Donaldson, K.Y. and Hasselman, D.P.H. 1990 Role of interfacial thermal barrier in the effective thermal diffusivity/conductivity of $\mathrm{SiC}$-fiber-reinforced reaction-bounded silicon nitride. J. Am. Ceram. Soc. 73, 312-31 6 .

Bruno, O. 1991 The effective conductivity of strongly heterogeneous composites. Proc. R. Soc. Lond. A. 433, 353-381.

Chiew, Y.C. and Glandt, E.D. 1987 Effective conductivity of dispersions: the effect of resistance at the particle surfaces. Chem. Eng. Sci 42, 2677-2685.

Every, A.G., Tzou, Y., Hasselman, D.P.H. and Ray, R. 1992 The effect of particle size on the thermal conductivity of ZnS/diamond composites. Acta Metall. Matter 40, 123-129.

Garnet, K.W. and Rosenberg, H.M. (1974) The thermal conductivity of epoxy-resin (powder composite materials. J. Phys. D. Appl. Phys 7, 1247-1258.

Golden, K. and Papanicolaou, G. 1983 Bounds for effective parameters of heterogeneous media by analytic continuation. Comm. Math. Phys. 90, 473.

Hashin, Z. 1992 Extremum principles for elastic heterogeneous media with imperfect interfaces and their application to bounding of effective moduli. J. Mech. Phys. Solids 40, 767-781.

Hashin, Z. and Shtrikman, S. 1962 A variational approach to the theory of the effective magnetic permeability of multiphase materials. J. Appl. Phys. 33, 3125-3131.

Hasselman, D.P.H., Donaldson, K.Y. and Thomas, J.R. Jr. 1993 Effective thermal conductivity of uniaxial composite with cylindrically orthotropic carbon fibers and interfacial thermal barrier. J. of Composite Materials 27, 637-644.

Hasselman, D.P.H. and Johnson, L.F. 1987 Effective thermal conductivity of composites with interfacial thermal barrier resistance. J. of Composite Materials 21, 508-515.

Latour, L.L., Svoboda, K., Mitra, P.O. and Sotak, C.H. 1994 Time-dependent diffusion of water in a biological model system. (to appear). 
Lenè, F. and Leguillon, D. 1982 Homogenized constitutive law for a partially cohesive composite material. Int. J. Solids Structures 18, 413-458.

Lipton, R. and Vernescu, B. 1995 Variational principles and bounds for elastic composites with interfacial slip. forthcoming.

Lurie, K.A. and Cherkaev, A.V. 1986 Effective characteristic of composite materials and the optimal design of structural elements. (in Russian) Uspekhi Mekhaniki 9 Advances in Mechanics.

Maxwell, J. C. 1904 A Treatise on Elasticity and Magnetism, vol.1, Oxford: Pergamon Press.

Milton, G. W. and Kohn, R. V. 1988 Variational bounds on the effective moduli of anisotropic composites J. Mech. Phys. Solids 36, 597-629.

Murat, F. and Tartar, L. 1989 Calcul des Variations et homogénéisation, in Les Methodes de 1'Homogénéisation: Théorie et Applications en Physique Coll. de la Dir. des Etudes et Reserches de Electr. del France; Eyrolles, Paris pp. 319-370.

Rayleigh, Lord 1892 On the influence of obstacles arranged in rectangular order upon the properties of a medium. Philos. Mag. 34, 481-507

Torquato, S. and Rubinstein, J 1991 Improved bounds on the effective conductivity of high-contrast suspensions. J. Appl. Phys. 69, 7118-7125.

Willis, J. R. 1982 Elasticity theory of composites. In Mechanics of Solids (ed. H. G. Hopkins and M. J. Sewell) Oxford: Pergamon Press. 